\title{
Order Reduction of Parametrically Excited Nonlinear Systems: Techniques and Applications
}

\author{
S. C. SINHA ${ }^{1, *}$, SANGRAM REDKAR ${ }^{1}$, VENKATESH DESHMUKH ${ }^{2}$, and ERIC A. BUTCHER ${ }^{2}$ \\ ${ }^{1}$ Nonlinear Systems Research Laboratory, Department of Mechanical Engineering, Auburn University, Auburn, AL 36849, \\ U.S.A.; ${ }^{2}$ Department of Mechanical Engineering, University of Alaska Fairbanks, Fairbanks, AK 99775, U.S.A.; \\ *Author for correspondence (e-mail: ssinha@eng.auburn.edu; fax: +1-334-844-3370)
}

(Received: 5 November 2004; accepted: 16 November 2004)

\begin{abstract}
The basic problem of order reduction of nonlinear systems with time periodic coefficients is considered in state space and in direct second order (structural) form. In state space order reduction methods, the equations of motion are expressed as a set of first order equations and transformed using the Lyapunov-Floquet (L-F) transformation such that the linear parts of new set of equations are time invariant. At this stage, four order reduction methodologies, namely linear, nonlinear projection via singular perturbation, post-processing approach and invariant manifold technique, are suggested. The invariant manifold technique yields a unique 'reducibility condition' that provides the conditions under which an accurate nonlinear order reduction is possible. Unlike perturbation or averaging type approaches, the parametric excitation term is not assumed to be small. An alternate approach of deriving reduced order models in direct second order form is also presented. Here the system is converted into an equivalent second order nonlinear system with time invariant linear system matrices and periodically modulated nonlinearities via the L-F and other canonical transformations. Then a master-slave separation of degrees of freedom is used and a nonlinear relation between the slave coordinates and the master coordinates is constructed. This method yields the same 'reducibility conditions' obtained by invariant manifold approach in state space. Some examples are given to show potential applications to real problems using above mentioned methodologies. Order reduction possibilities and results for various cases including 'parametric', 'internal', 'true internal' and 'true combination resonances' are discussed. A generalization of these ideas to periodic-quasiperiodic systems is included and demonstrated by means of an example.
\end{abstract}

Key words: Lyapunov-Floquet transformation, nonlinear time periodic systems, order reduction

\section{Introduction}

Many structural systems are modeled using finite element or lumped parameter techniques. In this process, the dynamic problem is reduced to a large set of ordinary differential equations. These equations may be linear or nonlinear where nonlinearities can arise from geometry or material behavior. An important class of problems gives rise to a large number of equations with time periodic coefficients such as the dynamics of rotating systems, like helicopter blades, asymmetric rotor-bearing systems and structures subjected to periodic loadings, etc. For the purpose of modal analysis, control and model testing, however, only a few dominating modes are important. Therefore, the development of reduced order models that approximate the dynamics of original large-scale system constitute an important problem.

Several linear approaches have been proposed to construct reduced order models for time invariant systems (of dimension $r$ ) that approximate the dynamics of the actual higher order system of dimension $n$. The response of the reduced order model of dimension $r(\ll n)$ is equivalent to the original large-scale system in some desired sense [1]. One of the techniques that developed primarily within the discipline of finite element analysis is called the 'Guyan reduction' [2]. This is a linear static reduction procedure, which provides a reduced order model in structural form with coordinates that are a subset of the 


\section{S. C. Sinha et al.}

original coordinate system. Recently, 'Guyan reduction' has been modified to account for inertia as well as stiffness properties. Other linear reduction methods, such as the internal balancing technique, are accomplished in state space form and are more common to control applications [3]. Guyan-like methods may also be applied to nonlinear systems, in which case the order reduction transformation is correct only for the linear terms. While such a linear strategy may yield acceptable results, the effects of the nonlinear terms are neglected in the order reduction process.

In order to develop a nonlinear order reduction technique for nonlinear systems, the concept of nonlinear normal modes (NNMs) may be utilized. As suggested by Shaw and Pierre [4], the nonlinear normal modes are defined as motions occurring on invariant manifolds, which are generally tangent to the corresponding eigenvectors of the linearized system at the equilibrium position and can be obtained analytically in a series form by various techniques [5]. The invariance property ensures that any motion starting exactly in a given modal manifold will remain in that manifold. One may perform a nonlinear modal analysis in order to obtain the system response in terms of some nonlinear modal coordinates. Model reduction using the nonlinear modal coordinates is advantageous because one may use fewer nonlinear normal modes than linear ones to perform equally accurate modal analysis of nonlinear systems. The order reduction may be carried out in state space [6] or in second-order (structural) form [7]. In a recent paper, Burton and Rhee [8] suggested a NNM-based order reduction method in structural form and compared it with the linear reduction procedure for time invariant systems.

In most cases, the original large-scale system of equations contains constant coefficients and methods discussed above can be used for order reduction. Recently, order reduction techniques for linear systems with time periodic coefficients have been reported using approximate multipoint Krylov techniques and time-varying Padé approximation [9] from a control system perspective. In the area of structural dynamics of time-varying linear and nonlinear systems, very little work has been reported. In an isolated study [10], modal analysis and order reduction was performed on a rotor dynamic problem without taking into account the contribution of periodic terms. In general, such reduced models cannot portray the correct dynamics of the original periodic systems, to say the least. For a more meaningful approach, the contribution of periodic terms must be included in the transformation matrix. This may be accomplished by the use of Lyapunov-Floquet (L-F) transformation that converts the time varying linear system matrix into an equivalent time invariant form. The details of computation and application of $\mathrm{L}-\mathrm{F}$ transformation can be found in [11, 12]. Deshmukh et al. [13] recently applied this concept to develop an order reduction technique and control strategy for large-scale time periodic systems in state space form. Control laws based on modal analysis and aggregations were developed in the original coordinates by applying well-known control strategies to the reduced system in the transformed domain. A similar approach to nonlinear time periodic system may not yield acceptable results.

Very recently, Sinha et al. [14] have suggested that an order reduction is possible for nonlinear time periodic systems through an application of L-F transformation and invariant manifold concept. As in the case of 'time periodic center manifold theory', where 'stable' states are expressed as periodically modulated nonlinear functions of 'critical' states, they proposed to express the 'slave' (non-dominant) states as periodically-modulated nonlinear functions of the 'master' (dominant) states. This is a generalization of the idea suggested by Shaw and Pierre [6] for autonomous systems. The approach implies the existence of periodically-varying invariant manifolds for time-periodic dynamical systems.

A novel approach for order reduction of parametrically excited nonlinear structural systems represented by a set of second order equations using invariant manifolds is also presented in this work. It is the generalization of the approach presented by Burton and coworkers [7, 8] for autonomous systems. Here, the original set of second order time-periodic nonlinear equations is converted into a second order nonlinear system with time invariant linear system matrices and periodically modulated nonlinearities 
via the L-F and other canonical transformations. Then a master-slave separation of degrees of freedom is used and a nonlinear relation between the slave coordinates and the master coordinates is constructed [15]. In this process, closed form expressions for 'true internal' and 'true combination resonances' are obtained for various nonlinearities, as before. Reduced order description of system response obtained from models based on nonlinear order reduction techniques is expected to be more accurate than those obtained via linear methods.

Finally, the order reduction techniques in the state space can be generalized to reduce the order of a special class of problems known as periodic-quasiperiodic systems. These systems have a time periodic linear part and nonlinearities with quasiperiodic coefficients. The procedure for normal forms computation of these types of problems is suggested by E. G. Belaga as described by Arnold [16]. Very recently, Wooden demonstrated a practical way of computing the normal forms via L-F and quasiperiodic near identity transformations [17]. From an order reduction point of view, all approaches except the invariant manifold technique can be straightforwardly used. The invariant manifold approach can also be generalized for these problems by assuming a nonlinear quasiperiodic relationship between the 'master' and 'slave' states. This approach yields a 'generalized reducibility condition' that tells us whether (or not) an accurate order reduction is possible.

The paper is divided into eight sections. In the second section, the general order reduction procedure in state space is outlined. An example is presented in Section 3, which demonstrates the application of state space order reduction methodologies. Order reduction procedure in second order (structural) form is included in Section 4 and followed by an example in Section 5. Section 6 presents the generalization of order reduction ideas to periodic-quasiperiodic systems, with an illustrative example presented in Section 7. Discussion and conclusions are included in Section 8.

\section{Model Order Reduction Techniques in State Space}

Consider the linear periodic system

$$
\dot{\mathbf{x}}(t)=\mathbf{A}(t) \mathbf{x}(t), \quad \mathbf{A}(t+T)=\mathbf{A}(t)
$$

where $\mathbf{x}(t)$ is an $n$ vector and $\mathbf{A}(t)$ is an $n \times n$ periodic matrix with principal period $T$. The state transition matrix (STM) $\boldsymbol{\Phi}(t)$ of Equation (1) can be factored as [18]

$$
\mathbf{\Phi}(t)=\mathbf{Q}(t) e^{\mathbf{R} t}, \quad \mathbf{Q}(t)=\mathbf{Q}(t+2 T), \quad \mathbf{Q}(0)=\mathbf{I}
$$

where the $n \times n$ matrix $\mathbf{Q}(t)$ is real and periodic with period $2 T, \mathbf{R}$ is an $n \times n$ real time invariant matrix whose eigenvalues (Floquet exponents) must lie in the left-half plane for (asymptotic) stability, and $\mathbf{I}$ is the identity matrix. $\mathbf{Q}(t)$ is known as the Lyapunov-Floquet (L-F) transformation matrix [11] and the transformation $\mathbf{x}(t)=\mathbf{Q}(t) \mathbf{z}(t)$ produces a real time invariant representation given by

$$
\dot{\mathbf{z}}(t)=\mathbf{R z}(t)
$$

in which the linear system matrix $\mathbf{R}$ is time invariant as has been noted. 


\subsection{ORder REDUCtion USING the LineAR MEthod}

Now consider a system described by a set of $n$ nonlinear differential equations with time periodic coefficients in state space form as

$$
\dot{\mathbf{x}}(t)=\mathbf{A}(t) \mathbf{x}(t)+\mathbf{f}(\mathbf{x}, t)
$$

where $\mathbf{f}(\mathbf{x}, t)$ is a nonlinear T-periodic $n$ vector such that $\mathbf{f}(0, t)=0$.

Applying the L-F Transformation $\mathbf{x}(t)=\mathbf{Q}(t) \overline{\mathbf{y}}(t)$ produces

$$
\dot{\overline{\mathbf{y}}}(t)=\mathbf{R} \overline{\mathbf{y}}(t)+\mathbf{Q}^{-1}(t) \mathbf{f}(\overline{\mathbf{y}}, t)
$$

After the modal transformation, $\overline{\mathbf{y}}(t)=\mathbf{M z}(t)$, we have

$$
\dot{\mathbf{z}}(t)=\mathbf{J z}(t)+\mathbf{M}^{-1} \mathbf{Q}^{-1}(t) \mathbf{f}(\mathbf{z}, t) \equiv \mathbf{J z}(t)+\mathbf{w}(\mathbf{z}, t)
$$

where $\mathbf{J}$ is the Jordan form of $\mathbf{R}$ and $\mathbf{w}(\mathbf{z}, t)$ represents an appropriately defined nonlinear time-varying vector consisting of monomials of $z_{j}$. The objective of order reduction is to replace the nonlinear time periodic system described by Equation (6) by an equivalent system given by

$$
\dot{\mathbf{z}}_{r}(t)=\mathbf{J}_{r} \mathbf{z}_{r}(t)+\overline{\mathbf{w}}_{r}\left(\mathbf{z}_{r}, t\right)
$$

where $\mathbf{z}_{r}$ is an $r(r \ll n)$ vector of the dominant states to be retained, $\mathbf{J}_{r}$ is an $r \times r$ Jordan block corresponding to the dominant states and $\overline{\mathbf{w}}_{r}\left(\mathbf{z}_{r}, t\right)$ is a nonlinear vector function of appropriate dimensions consisting only of the dominant states.

At this stage Equation (6) may be partitioned as

$$
\left\{\begin{array}{c}
\dot{\mathbf{z}}_{r} \\
\dot{\mathbf{z}}_{s}
\end{array}\right\}=\left[\begin{array}{cc}
\mathbf{J}_{r} & 0 \\
0 & \mathbf{J}_{s}
\end{array}\right]\left\{\begin{array}{l}
\mathbf{z}_{r} \\
\mathbf{z}_{s}
\end{array}\right\}+\left\{\begin{array}{c}
\mathbf{w}_{r}\left(\mathbf{z}_{r}, \mathbf{z}_{s}, t\right) \\
\mathbf{w}_{s}\left(\mathbf{z}_{r}, \mathbf{z}_{s}, t\right)
\end{array}\right\}
$$

where $\mathbf{z}_{s}$ is an $(n-r)$ vector of non-dominant states, $\mathbf{J}_{s}$ is the Jordan block of dimension $(n-r) \times(n-r)$ corresponding to the non-dominant states and $\mathbf{w}_{r}\left(\mathbf{z}_{r}, \mathbf{z}_{s}, t\right)$ and $\mathbf{w}_{s}\left(\mathbf{z}_{r}, \mathbf{z}_{s}, t\right)$ consist of the monomials of $\mathbf{z}$ with periodic coefficients. In the linear approach, the contribution of the non-dominant states is considered insignificant and hence neglected. Thus, by neglecting Equation (8) and setting the nondominant states to zero $\left(\mathbf{z}_{s}=0\right)$, the entire system dynamics is approximated by

$$
\dot{\mathbf{z}}_{r}(t)=\mathbf{J}_{r} \mathbf{z}_{r}(t)+\mathbf{w}_{r}\left(\mathbf{z}_{r}, 0, t\right)
$$

Equation (9) is the reduced order model of the actual large-scale system described by Equation (6). Equation (9) can be integrated numerically and using the transformation $\mathbf{x}(t)=\mathbf{Q}(t) \mathbf{M T} \mathbf{z}_{r}(t)$, where $\mathbf{T}=\left[\begin{array}{lll}\mathbf{I}_{r \times r} & \mathbf{0}_{r \times(n-r)}\end{array}\right]^{\mathrm{T}}$, all the states in $\mathbf{x}$ can be recovered.

The linear technique is simple and easy to implement. However, it does not give a clear insight if the dynamics is complex and involves 'internal' and/or 'parametric resonance'. The approximation error is larger for larger amplitudes and sometimes the results can be misleading. 


\subsection{Order Reduction Using the Nonlinear Projection Via Singular Perturbation (SP)}

Once again, we consider Equation (4) which can be transformed to Equation (8). The objective here is to approximate Equation (8) by a reduced order system given by

$$
\dot{\mathbf{z}}_{r}(t)=\mathbf{J}_{r} \mathbf{z}_{r}(t)+\mathbf{w}_{r}\left(\mathbf{z}_{r}, \psi\left(\mathbf{z}_{r}, t\right), t\right)
$$

where $\mathbf{z}_{r}(t), \mathbf{J}_{r}, \mathbf{w}_{r}\left(\mathbf{z}_{r}, t\right)$ are as defined before and $\psi\left(\mathbf{z}_{r}, t\right)$ is a nonlinear time periodic operator relating non-dominant states to dominant states, i.e.,

$$
\mathbf{z}_{S}(t)=\psi\left(\mathbf{z}_{r}, t\right)
$$

In order to determine this relationship, since $\mathbf{z}_{s}(t)$ are non-dominant states, we rewrite Equation (8) as a classical singular perturbation problem [19]. We partition the system dynamics into slow and fast dynamics by ordering it according to the decay rates (or system eigenvalues) as

$$
\begin{array}{r}
\dot{\mathbf{z}}_{r}=\mathbf{J}_{r} \mathbf{z}_{r}+\mathbf{w}_{r}\left(\mathbf{z}_{r}, \mathbf{z}_{s}, t\right) \\
\varepsilon \dot{\mathbf{z}}_{s}=\mathbf{J}_{s} \mathbf{z}_{s}+\mathbf{w}_{s}\left(\mathbf{z}_{r}, \mathbf{z}_{s}, t\right)
\end{array}
$$

setting $\varepsilon=0$, we have

$$
\begin{array}{r}
\dot{\mathbf{z}}_{r}=\mathbf{J}_{r} \mathbf{z}_{r}+\mathbf{w}_{r}\left(\mathbf{z}_{r}, \mathbf{z}_{s}, t\right) \\
0=\mathbf{J}_{s} \mathbf{z}_{s}+\mathbf{w}_{s}\left(\mathbf{z}_{r}, \mathbf{z}_{s}, t\right)
\end{array}
$$

Thus, the system of differential equations given by Equation (8) is converted into a Differential Algebraic System (DAEs) which is a reduced order approximation of the original large-scale system. One may solve the DAE system given by Equation (13) numerically and using the transformation $\mathbf{x}(t)=\mathbf{Q}(t) \mathbf{M z}(t)$ recover all the states in $\mathbf{x}$. However, in many cases one can find $\psi\left(\mathbf{z}_{r}, t\right)$ (cf. Equation (11)) approximately by solving Equation (13b) as follows.

Using a fixed-point iteration approach [20-23], $\mathbf{z}_{s}$ can be approximated as

$$
\mathbf{z}_{s}=-\mathbf{J}_{s}^{-1} \mathbf{w}_{s}\left(\mathbf{z}_{r}, 0, t\right) \equiv \psi_{1}\left(\mathbf{z}_{r}, t\right)
$$

Substituting Equation (14) into Equation (8), the reduced order Equation can be written as

$$
\dot{\mathbf{z}}_{r}(t)=\mathbf{J}_{r} \mathbf{z}_{r}(t)+\mathbf{w}_{r}\left(\mathbf{z}_{r}, \psi_{1}\left(\mathbf{z}_{r}, t\right), t\right)
$$

If one uses two fixed-point iterations [20] as

$$
\begin{aligned}
\mathbf{z}_{s_{1}} & =-\mathbf{J}_{s}^{-1} \mathbf{w}_{s}\left(\mathbf{z}_{r}, 0, t\right) \\
\mathbf{z}_{s} & =-\mathbf{J}_{s}^{-1} \mathbf{w}_{s}\left(\mathbf{z}_{r}, \mathbf{z}_{s_{1}}, t\right) \equiv \boldsymbol{\psi}_{2}\left(\mathbf{z}_{r}, t\right)
\end{aligned}
$$

then Equations (17) and (8) yield the reduced order Equation as

$$
\dot{\mathbf{z}}_{r}(t)=\mathbf{J}_{r} \mathbf{z}_{r}(t)+\mathbf{w}_{r}\left(\mathbf{z}_{r}, \boldsymbol{\psi}_{2}\left(\mathbf{z}_{r}, t\right), t\right)
$$




\section{S. C. Sinha et al.}

As before, Equation (15) or (18) can be integrated numerically and using the L-F and the modal transformations all the states in $\mathbf{x}$ can be recovered. The nonlinear projection method yields a 'non-flat' sub-manifold and it is expected to yield better results than its linear counterpart.

\subsection{Order Reduction Using the Post-Processing Method}

This technique minimizes the computational cost associated with the nonlinear projection method. It has been shown [24] that this approach is often as accurate as the 'nonlinear projection technique' and requires less computational effort. The procedure remains the same as described in Section 2.1 up to the partitioned form given by Equation (8).

At this stage, it is assumed that

$$
\mathbf{z}_{r}=\mathbf{z}_{r p}+\kappa\left(\mathbf{z}_{\bar{r}}, t\right)
$$

where $\mathbf{z}_{r p}$ is the response obtained by integrating Equation (9) (which is the reduced order model obtained using the linear approach) and $\kappa\left(\mathbf{z}_{\bar{r}}, t\right)$ is the post-processing correcting factor, which uplifts the 'flat' manifold [20]. This correcting factor can be obtained by solving Equation (8) using iterative schemes. The main advantage here is that the computational effort associated with integrating Equation (7) is much less than Equation (15) or (18).

\subsection{ORDER REDUCTION Using THE TIME PERIOdic InVARIANT MANIFOLD}

This methodology is based on the concept of invariant manifold for time-periodic systems. It implies that there exists a nonlinear time periodic relationship between dominant and non-dominant states, and therefore it is possible to reduce a large-scale system to a smaller system represented only by the dominant (master) states.

Consider the nonlinear time periodic system given by Equation (8). In this approach, a nonlinear relationship is assumed between the non-dominant $\left(\mathbf{z}_{S}\right)$ and dominant $\left(\mathbf{z}_{r}\right)$ states as

$$
\mathbf{z}_{s}=\sum_{i} \mathbf{h}_{i}\left(\mathbf{z}_{r}, t\right) \equiv \mathbf{H}\left(\mathbf{z}_{r}, t\right)
$$

where

$$
\mathbf{h}_{i}=\sum_{\overline{\mathbf{m}}} \overline{\mathbf{h}}_{i}(t) \mathbf{z}_{1}^{m_{1}} \ldots \mathbf{z}_{r}^{m_{r}}, \quad \overline{\mathbf{m}}=\left(m_{1}, \ldots, m_{r}\right)^{\mathrm{T}}, \quad m_{1}+\cdots+m_{r}=i, \quad i=2,3, \ldots
$$

Here $\overline{\mathbf{h}}_{i}(t)$ are the unknown periodic vector coefficients or order $i$ with period $2 T$. Equation (20) represents a periodically-varying manifold in the $\mathbf{x}$ and $\mathbf{z}$ coordinate systems. Substitution of Equation (20) into Equation (8) yields

$$
\frac{\partial \mathbf{H}}{\partial t}+\frac{\partial \mathbf{H}}{\partial \mathbf{z}_{r}}\left(\mathbf{J}_{r} \mathbf{z}_{r}+\mathbf{w}_{r}\left(\mathbf{z}_{r}, \mathbf{H}, t\right)\right)=\mathbf{J}_{s} \mathbf{H}+\mathbf{w}_{s}\left(\mathbf{z}_{r}, \mathbf{H}, t\right)
$$

At this point, it should be observed that, if we equate terms of the same order $i$ in the above Equation, then we can solve

$$
\frac{\partial \mathbf{h}_{i}}{\partial t}+\frac{\partial \mathbf{h}_{i}}{\partial \mathbf{z}_{r}} \mathbf{J}_{r} \mathbf{z}_{r}-\mathbf{J}_{s} \mathbf{h}_{i}=\mathbf{w}_{s i}-\left(\frac{\partial \mathbf{H}}{\partial \mathbf{z}_{r}} \mathbf{w}_{r}\right)_{i} \text { for } i=2,3,4, \ldots
$$


sequentially for $\mathbf{h}_{i}$ beginning with the quadratic terms where $\mathbf{w}_{s i}$ contains the $i$ th order terms of $\mathbf{w}_{s}$. From Equation (20) the second term on the right hand side of Equation (22) can be written as

$$
\left(\frac{\partial \mathbf{H}}{\partial \mathbf{z}_{r}} \mathbf{w}_{r}\right)_{i} \equiv \frac{\partial \mathbf{h}_{i-1}}{\partial \mathbf{z}_{r}} \mathbf{w}_{r 2}+\frac{\partial \mathbf{h}_{i-2}}{\partial \mathbf{z}_{r}} \mathbf{w}_{r 3}+\ldots
$$

where $\mathbf{w}_{r i}$ contains the $i$ th order terms of $\mathbf{w}_{r}$. This implies that for $i=2$, all the terms in Equation (23) are zero. For $i=3$, the second term is zero while for $i=4$; the third term is zero and so on.

In order to solve this partial differential Equation approximately, we expand the known and the unknown periodic coefficients into 2T-periodic Fourier series as

$$
\begin{aligned}
& \mathbf{h}_{i}\left(\mathbf{z}_{r}, t\right)=\sum_{j=1}^{s} \sum_{\overline{\mathbf{m}}} \sum_{\nu=-\infty}^{\infty} h_{j \bar{m} \nu} e^{\frac{i \nu \pi t t}{T}}\left|\mathbf{z}_{r}\right|^{\mathbf{m}} e_{j} \\
& \mathbf{w}_{s i}\left(\mathbf{z}_{r}, t\right)-\left(\frac{\partial \mathbf{H}}{\partial \mathbf{z}_{r}} \mathbf{w}_{r}\right)_{i}=\sum_{j=1}^{s} \sum_{\overline{\mathbf{m}}} \sum_{\nu=-\infty}^{\infty} a_{j \bar{m} \nu} e^{\frac{i \bar{v} t t}{T}}\left|\mathbf{z}_{r}\right|^{\mathbf{m}} e_{j}
\end{aligned}
$$

where $\left|\mathbf{z}_{r}\right|=z_{1}^{m_{1}} z_{2}^{m_{2}} \ldots z_{r}^{m_{r}}, \bar{i}=\sqrt{-1}, \quad m_{1}+\cdots+m_{r}=i ; i=2,3, \ldots, a_{j \bar{m} v}$ are the known Fourier coefficients of the periodic functions, whereas $h_{j \bar{m} \nu}$ are the unknown Fourier coefficients of the manifold relation and $e_{j}$ is the $j$ th member of the natural basis.

A term-by-term comparison of the Fourier coefficients yields

$$
h_{j m \nu}=\frac{a_{j m \nu}}{\frac{i v \pi}{T}+\sum_{l=1}^{r}\left(m_{l} \lambda_{l}\right)-\bar{\lambda}_{p}}
$$

where $\lambda_{1}, \lambda_{2}, \ldots, \lambda_{r}$ are the characteristic exponents of the Jordan matrix $\mathbf{J}_{r}$ and $\bar{\lambda}_{p} ; p=$ $1,2, \ldots, s$ are the characteristic exponents of $\mathbf{J}_{s}$. Therefore, the 'reducibility condition' is expressed as

$$
\frac{\bar{i} \nu \pi}{T}+\sum_{l=1}^{r}\left(m_{l} \lambda_{l}\right)-\bar{\lambda}_{p} \neq 0, \quad \forall v=0, \pm 1, \pm 2, \ldots ; \quad p=1,2, \ldots, s .
$$

It is obvious that if the 'reducibility condition' is satisfied then the vector $\mathbf{H}\left(\mathbf{z}_{r}, t\right)$ can be obtained and the 'slave' states can be expressed in terms of the 'master' states. However, when this condition is not satisfied, such a reduction is not possible and the 'slave' coordinates cannot be expressed as functions of the 'master' coordinates.

For $v=0$, a resonance occurs when a special linear combination of the $\lambda_{l}$ (characteristic exponents of the 'master' states) adds up to one of the $\bar{\lambda}_{p}$ (characteristic exponents of the 'slave' states). This may be referred to as the 'true internal resonance'. If $\mathbf{A}(t)$ in Equation (4) is time-invariant and derived from an autonomous Hamiltonian system (as would be the case for undamped vibration problems) then in Equation (27) the eigenvalues $\lambda_{n}= \pm \bar{i} \omega_{n}$, where $\omega_{n}$ are the natural frequencies of the linear system and the conditions for the conventional 'internal resonance', widely discussed in the literature, are recovered. Shaw et al. [6] derived these conditions for the special case of quadratic and cubic nonlinearities using Mathematica ${ }^{\mathrm{TM}}$. It can be easily verified that their result is a subset of the results contained in Equation (27).

For $v \neq 0$, the denominator in Equation (26) goes to zero when certain linear combination of $\bar{i} \omega / 2$ (where $\omega(=2 \pi / T)$ is the parametric excitation frequency) and the characteristic exponents of the 'master' states adds up to one of the characteristic exponents of the 'slave' states. This situation 


\section{S. C. Sinha et al.}

is referred to as the 'true combination resonance' case. Further, once again we look at a special form of Equation (4) such that $\boldsymbol{A}(t)=\mathbf{A}_{0}+\varepsilon \mathbf{A}_{1}(t)$, where $\mathbf{A}_{0}$ is derived from an autonomous Hamiltonian system. For $\varepsilon=0$ the eigenvalues of $\mathbf{A}_{0}$ are $\pm \bar{i} \omega_{n}$, where $\omega_{n}$ being the natural frequencies. It is interesting to observe here as $\varepsilon \rightarrow 0$ characteristic exponents $\lambda_{n} \rightarrow \pm \bar{i} \omega_{n}$, and from Equation (26) we recover the condition for conventional 'combination resonance' in parametrically excited nonlinear systems with a small parameter [25, 26]. The concept of 'parametric resonance' comes from the stability analysis of linear systems with time periodic coefficients. For a single degree of freedom system, the repeated multipliers have to be real and repeated as -1 or +1 . This implies that at least one pair of eigenvalues of the $\mathbf{J}$ matrix are a zero. For multiple degrees of freedom systems, one other possible situation could be that two pairs of Floquet exponents are purely imaginary and repeated. For systems with small parametric excitation terms (as discussed above), it takes the form of sum and difference type of resonance conditions. The details may be found in [27]. If the $\mathbf{J}$ matrix contains a pair of zeros (critical eigenvalues), then the order reduction process necessarily boils down to finding a time-periodic center manifold relation between the 'slave' and the 'master' states [28]. Since Floquet multipliers are either -1 or +1 , the system undergoes a 'flip' or a symmetry breaking (or transcritical) bifurcation, respectively. Once $\mathbf{H}\left(\mathbf{w}_{r}, t\right)$ has been determined, we obtain the Equation for the 'master' states $\mathbf{z}_{r}$ from Equation (8) as

$$
\dot{\mathbf{z}}_{r}=\mathbf{J}_{r} \mathbf{z}_{r}+\overline{\mathbf{w}}_{r}\left(\mathbf{z}_{r}, \mathbf{H}\left(\mathbf{z}_{r}, t\right), t\right)
$$

This is the reduced order system in the $\mathbf{z}$ domain. Now we can make use of the L-F transformation matrix $\mathbf{Q}(t)$ and the modal matrix $\mathbf{M}$ to map the results back to the original domain as $\mathbf{x}(t)=\mathbf{Q}(t) \mathbf{M} \overline{\mathbf{H}}\left(\mathbf{z}_{r}, t\right)$ where, $\overline{\mathbf{H}}=\left(\begin{array}{ll}\mathbf{z}_{r}^{\mathrm{T}} & \mathbf{H}^{\mathrm{T}}\left(\mathbf{z}_{r}, t\right)\end{array}\right)^{\mathrm{T}}$. Since this transformation is a Lyapunov transformation, stability properties are preserved and the existence of the invariant manifold is guaranteed. It is to be noted that, although the manifold itself varies periodically (in $\mathbf{x}$ or $\mathbf{z}$ coordinates), motion on this manifold is generally quasiperiodic in either coordinate system.

\section{An Application}

The techniques proposed in Section 2 can be effectively applied to reduce the order of practical engineering problems modeled by nonlinear differential systems with time periodic coefficients. As an illustration, we reduce the order of a system consisting of two inverted coupled pendulums moving in the horizontal plane with time-dependent load acting on each pendulum. Each pendulum is supported at the base by a torsional spring. The loading consists of time periodic concentrated axial loads. A diagram of the system is shown in Figure 1. The equations of motion expanded through cubic terms about the fixed point $\left(\theta_{1}, \theta_{2}, \dot{\theta}_{1}, \dot{\theta}_{2}\right)=(0,0,0,0)$ can be shown to be

$$
\begin{aligned}
\ddot{\theta}_{1} & +\frac{k_{t 1}}{m l^{2}} \theta_{1}+\frac{k}{4 m}\left[c_{1}\left(\theta_{1}-\theta_{2}\right)+c_{2}\left(\theta_{1}-\theta_{2}\right)^{2}+c_{3}\left(\theta_{1}-\theta_{2}\right)^{3}\right] \\
- & {\left[\frac{P_{11} l}{m l^{2}}+\frac{P_{12} l}{m l^{2}} \cos (\omega t)\right]\left(\theta_{1}-\frac{\theta_{1}^{3}}{6}\right)=0 } \\
\ddot{\theta}_{2} & +\frac{k_{t 2}}{m l^{2}} \theta_{2}+\frac{k}{4 m}\left[c_{1}\left(\theta_{2}-\theta_{1}\right)+c_{2}\left(\theta_{2}-\theta_{1}\right)^{2}+c_{3}\left(\theta_{2}-\theta_{1}\right)^{3}\right] \\
& -\left[\frac{P_{21} l}{m l^{2}}+\frac{P_{22} l}{m l^{2}} \cos (\omega t)\right]\left(\theta_{2}-\frac{\theta_{2}^{3}}{6}\right)=0
\end{aligned}
$$




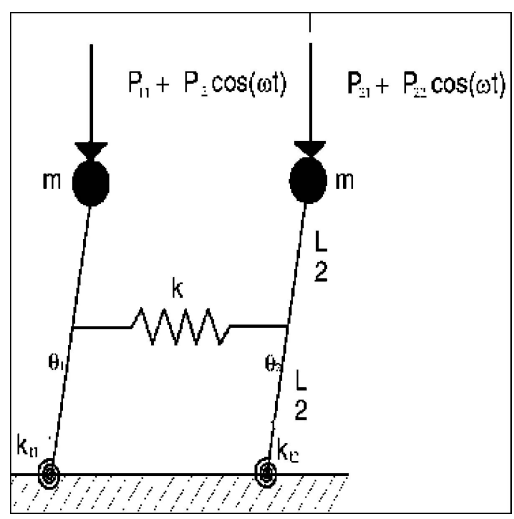

Figure 1. Coupled pendulums.

where $m, l, k_{t}$ and $k$ denote mass, length, torsional stiffness and coupling stiffness, respectively, and $c_{1}, c_{2}, c_{3}$ are coupling parameters. For simplicity, setting $P_{11}$ and $P_{21}$ equal to zero, Equation (29) can be rewritten in state space form as

$$
\begin{aligned}
\left\{\begin{array}{c}
\dot{x}_{1} \\
\dot{x}_{2} \\
\dot{x}_{3} \\
\dot{x}_{4}
\end{array}\right\}= & {\left[\begin{array}{cccc}
0 & 0 & 1 & 0 \\
0 & 0 & 0 & 1 \\
-\left(\omega_{n_{1}}^{2}-\varepsilon p_{1} \cos (\omega t)\right) & b & 0 & 0 \\
b & -\left(\omega_{n_{2}}^{2}-\varepsilon p_{2} \cos (\omega t)\right) & 0 & 0
\end{array}\right]\left\{\begin{array}{l}
x_{1} \\
x_{2} \\
x_{3} \\
x_{4}
\end{array}\right\} } \\
& +\left\{\begin{array}{c}
0 \\
-\varepsilon p_{1} \cos (\omega t) \frac{x_{1}^{3}}{6}+c\left(x_{1}-x_{2}\right)^{2}+d\left(x_{1}-x_{2}\right)^{3} \\
-\varepsilon p_{2} \cos (\omega t) \frac{x_{2}^{3}}{6}-c\left(x_{1}-x_{2}\right)^{2}-d\left(x_{1}-x_{2}\right)^{3}
\end{array}\right\}
\end{aligned}
$$

where

$$
\begin{aligned}
\omega_{n_{1}}^{2} & =\left[\frac{k_{t 1}}{m l^{2}}+\frac{k}{4 m} c_{1}\right], \quad \omega_{n_{2}}^{2}=\left[\frac{k_{t 2}}{m l^{2}}+\frac{k}{4 m} c_{1}\right], \quad \varepsilon p_{1}=\frac{P_{12} l}{m l^{2}}, \quad \varepsilon p_{2}=\frac{P_{22} l}{m l^{2}}, \quad \omega=2 \pi, \\
b & =\frac{k}{4 m} c_{1}, \quad c=\frac{k}{4 m} c_{2}, \quad d=\frac{k}{4 m} c_{3},\left\{\begin{array}{lllll}
x_{1} & x_{2} & x_{3} & x_{4}
\end{array}\right\}^{\mathrm{T}}=\left\{\begin{array}{llll}
\theta_{1} & \theta_{2} & \dot{\theta}_{1} & \dot{\theta}_{2}
\end{array}\right\}^{\mathrm{T}}
\end{aligned}
$$

Applying the L-F and modal transformations $\mathbf{x}=\mathbf{Q}(t) \mathbf{M z}$, Equation (30) takes the form of Equation (6). By choosing a suitable set of values of coupling parameters $(b, c, d)$, the system can be made to undergo 'parametric resonance', (3:1 and 2:1), 'internal resonance', 'true internal resonance' or 'true combination resonance' [14]. These resonances are discussed in the context of this example.

\subsection{No RESONANCE OF ANY Kind}

The system parameters are chosen such that the system does not exhibit 'internal resonance', 'true internal resonance' or 'true combination resonance'. These parameters are given in Table 1. For this 
Table 1. Parameter set for the case when no parametric/internal resonance exists.

\begin{tabular}{lllllllll}
\hline Parameter & $\omega_{n_{1}}^{2}$ & $\omega_{n_{2}}^{2}$ & $\varepsilon p_{1}$ & $\varepsilon p_{2}$ & $\omega$ & $b$ & $c$ & $d$ \\
\hline Value & 3.5 & 5.5 & 5 & 5 & $2 \pi$ & 0.5 & 0 & 1 \\
\hline
\end{tabular}

set of parameters, the Floquet multipliers are given as $(-0.51 \pm 0.85 \bar{i},-0.88 \pm 0.45 \bar{i})$. The $\mathrm{L}-\mathrm{F}$ transformation matrix $\mathbf{Q}(t)$ is computed from the linear part of Equation (30) by the method suggested by Sinha et al. [11]. After using the modal transformation, Equation (6) for this case is given by

$$
\left\{\begin{array}{l}
\dot{z}_{1} \\
\dot{z}_{2} \\
\dot{z}_{3} \\
\dot{z}_{4}
\end{array}\right\}=\left[\begin{array}{cccc}
0 & -0.47 & 0 & 0 \\
0.47 & 0 & 0 & 0 \\
0 & 0 & 0 & -1.03 \\
0 & 0 & 1.03 & 0
\end{array}\right]\left\{\begin{array}{l}
z_{1} \\
z_{2} \\
z_{3} \\
z_{4}
\end{array}\right\}+\left\{\begin{array}{l}
w_{1}(\mathbf{z}, t) \\
w_{2}(\mathbf{z}, t) \\
w_{3}(\mathbf{z}, t) \\
w_{4}(\mathbf{z}, t)
\end{array}\right\}
$$

The Floquet exponents (eigenvalues of $\mathbf{R}$ matrix) are $( \pm 0.47 \bar{i}, \pm 1.03 \bar{i})$. It can be easily verified that the ratio of the angles of Floquet multipliers (or the ratio of Floquet exponents) for this set is 2.19, which implies they are not in 'true internal resonance' and the 'reducibility condition' given by Equation (27) is satisfied. $w_{i}(\mathbf{z}, t)$ are nonlinear functions with periodic coefficients comprising of all the states. Expressions for $w_{i}(\mathbf{z}, t)$ are obtained using Mathematica ${ }^{\mathrm{TM}}$ and are omitted here for brevity since these are quite long. At this stage, we reduce the order of the system given by Equation (31) using methods described in Section 2.

\subsubsection{Order Reduction Using the Linear Method}

Equation (31) comprises of 4 states, viz., $\left\{\begin{array}{lllll}z_{1} & z_{2} & z_{3} & z_{4}\end{array}\right\}^{\mathrm{T}}$. We choose $\mathbf{z}_{r}=\left\{\begin{array}{ll}z_{1} & z_{2}\end{array}\right\}^{\mathrm{T}}$ as the dominant states, as they correspond to smaller linear frequencies of the system. Following the procedure outlined in Section 2.1, we neglect the contribution from the non-dominant states $\mathbf{z}_{s}=\left\{\begin{array}{ll}z_{3} & z_{4}\end{array}\right\}^{\mathrm{T}}$ and the system dynamics is approximated by

$$
\left\{\begin{array}{l}
\dot{z}_{1} \\
\dot{z}_{2}
\end{array}\right\}=\left[\begin{array}{cc}
0 & -0.47 \\
0.47 & 0
\end{array}\right]\left\{\begin{array}{l}
z_{1} \\
z_{2}
\end{array}\right\}+\left\{\begin{array}{l}
\underline{w}_{1}\left(z_{1}, z_{2}, 0,0, t\right) \\
\underline{w}_{2}\left(z_{1}, z_{2}, 0,0, t\right)
\end{array}\right\}
$$

Equation (32) is the reduced order model of the system described by Equation (31). This reduced order system is integrated numerically and all states in $\mathbf{x}=\left\{\begin{array}{llll}\theta_{1} & \theta_{2} & \dot{\theta}_{1} & \dot{\theta}_{2}\end{array}\right\}^{\mathrm{T}}$ are obtained using the L-F and modal transformations. In Figure 2(a), the time trace of $\dot{\theta}_{1}$ obtained using this procedure is compared with the time trace of $\dot{\theta}_{1}$ obtained by integrating the original Equation (31). It can be seen that these time traces are similar but do not match accurately. In order to show the long time behavior, we simulate the reduced order model and the large-scale system for relatively longer period of time and plot the Poincaré maps (for both) sampled at the time period $(T=2 \pi / \omega)$ of parametric excitation. The Poincaré map of the large-scale system is shown in Figure 3a, while Figure 3b shows the Poincare map of the reduced system using the linear method. We observe that the Poincare map of the large-scale system shows a 'band' implying the existence of a complex quasiperiodic motion that is absent in the Poincaré map of the reduced system. However, the amplitudes are quite comparable. Thus, we may conclude that the reduced model based on the linear method captures the amplitude but does not capture the frequency content accurately for the given initial conditions. 


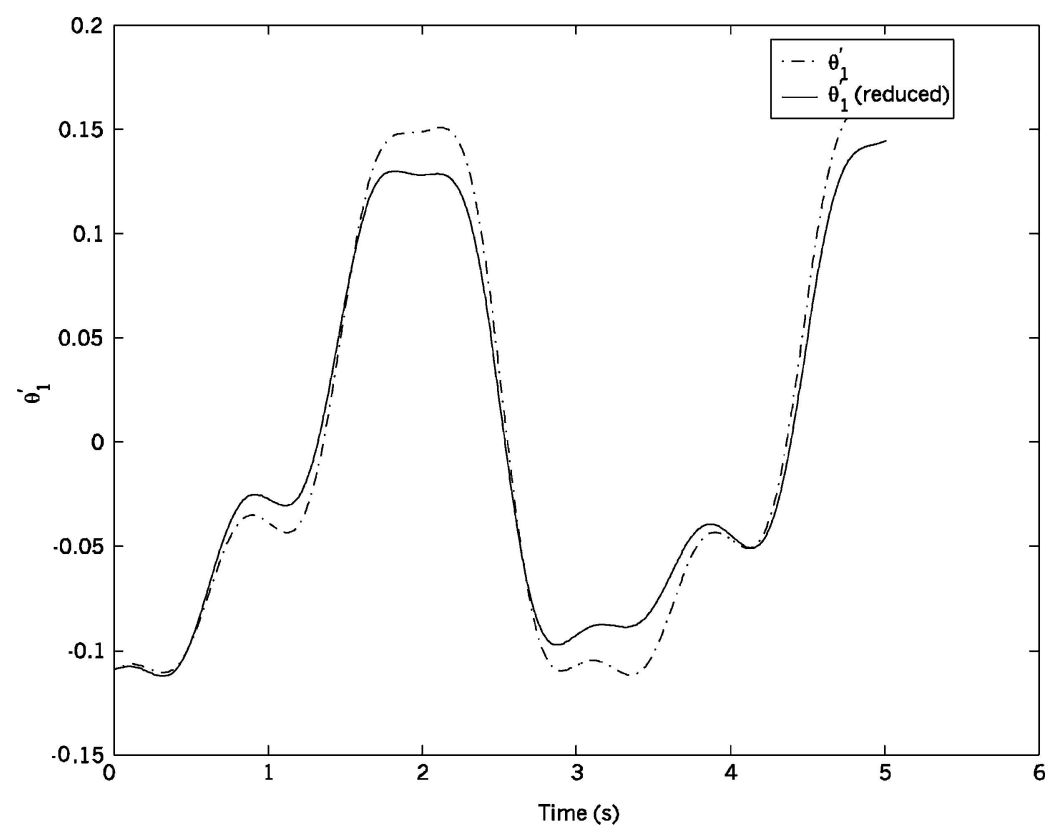

a) $\dot{\theta}_{1}-\dot{\theta}_{1(\text { linear method })}--$

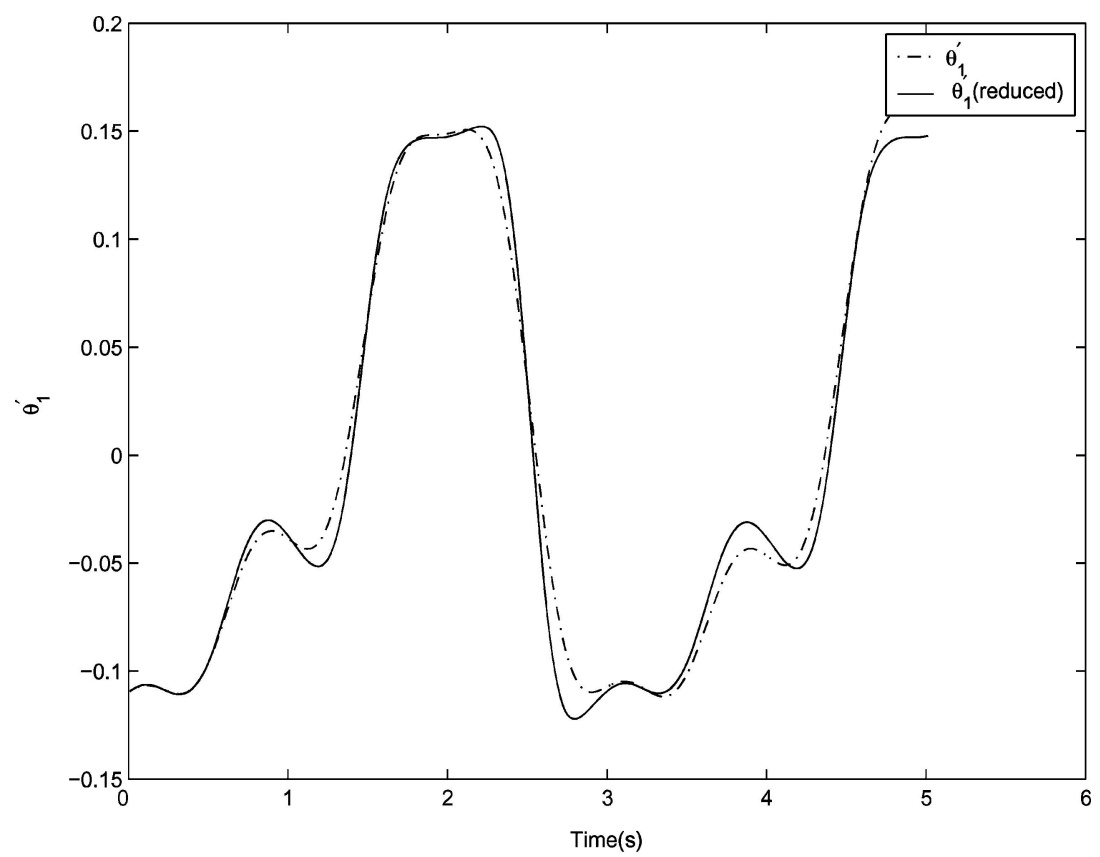

b) $\dot{\theta}_{1}-\dot{\theta}_{1 \text { (nonlinear DAE simulation) }}--$

Figure 2. (a) Time trace comparison for linear method. (b) Time trace comparison for nonlinear method based on singular Perturbation (SP) DAE solution. (c) Time trace comparison for nonlinear method based on singular perturbation (SP) fixed-point iteration. (d) Time trace comparison for nonlinear method based on the post-processing approach. (e) Time trace comparison for nonlinear method based on invariant manifold approach. 
248 S. C. Sinha et al.

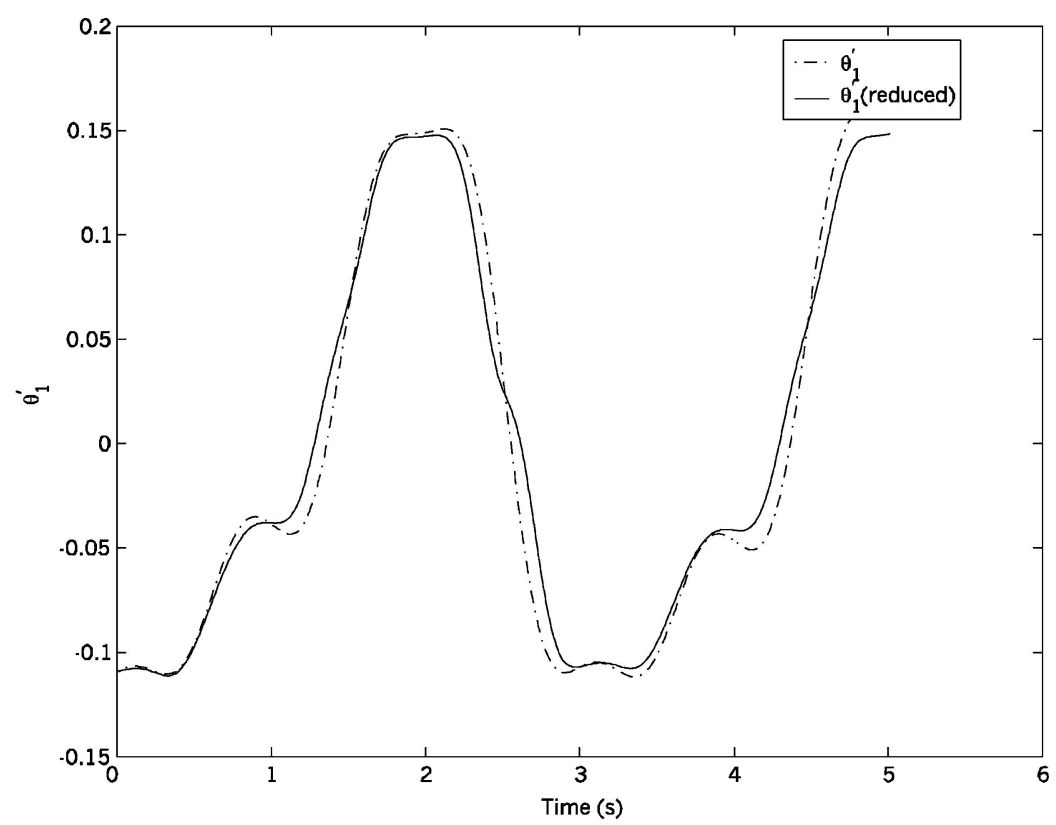

c) $\dot{\theta}_{1}-\dot{\theta}_{1 \text { (nonlinear projection) }}--$

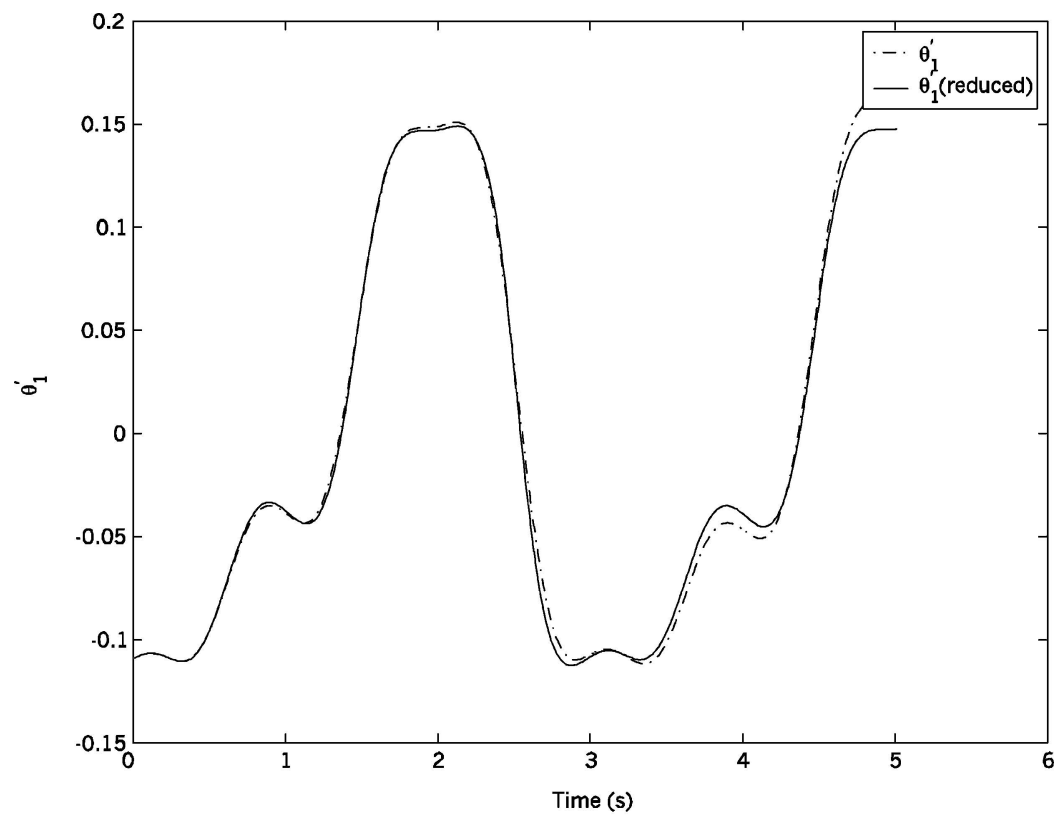

d) $\dot{\theta}_{1}-\dot{\theta}_{1(\text { nonlinear projection -postprocessed) }}--$

Figure 2. (Continued.) 


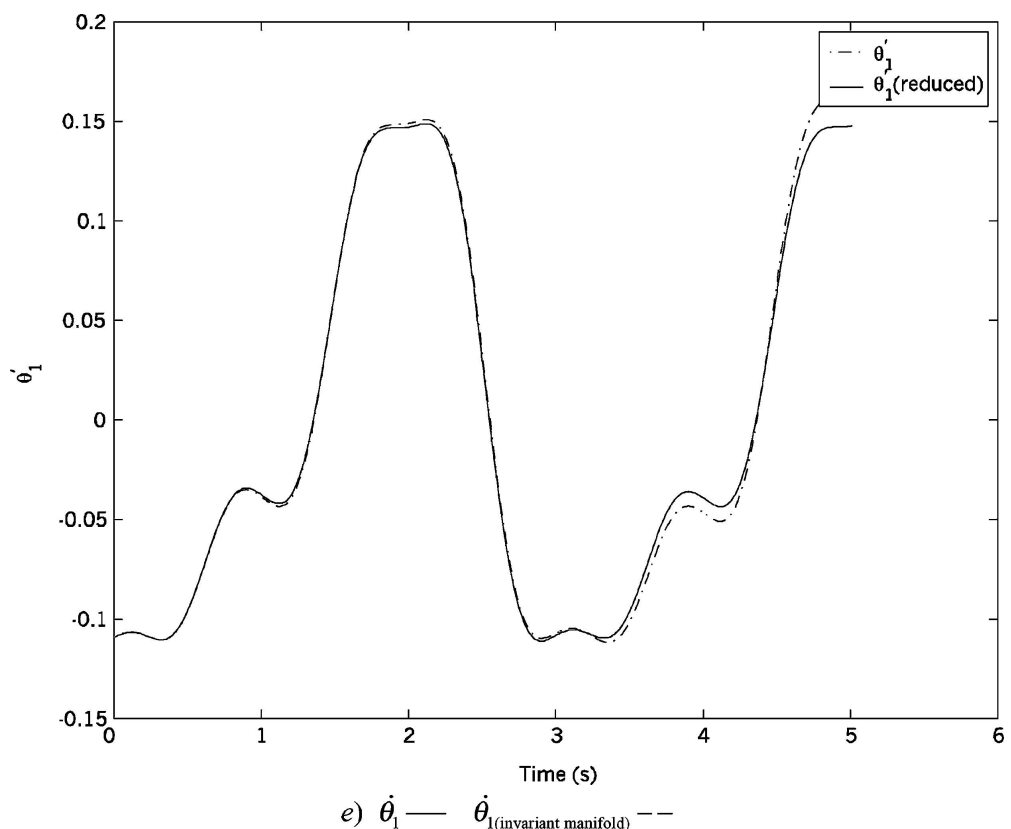

Figure 2. (Continued.)

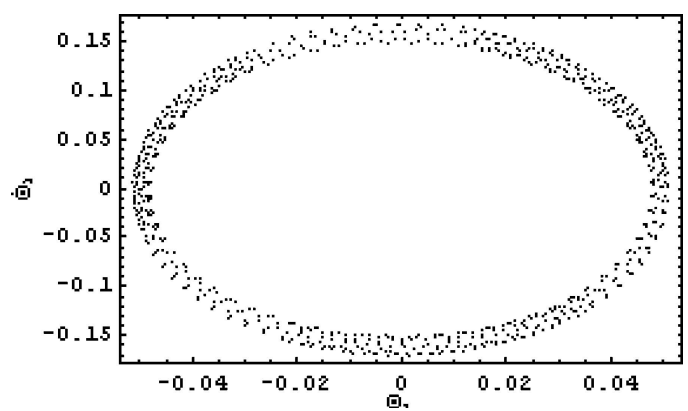

(a)

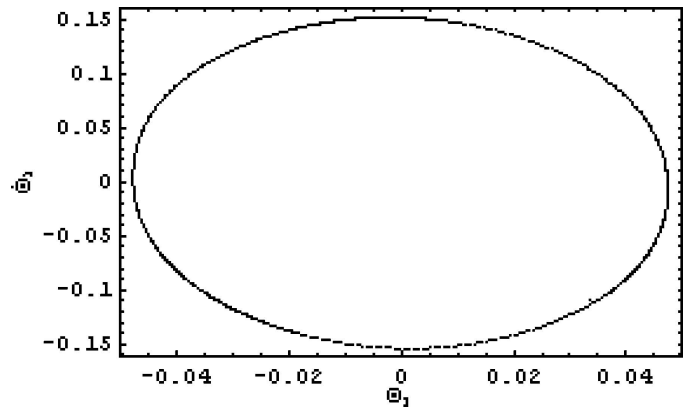

(b)

Figure 3. (a) Poincaré maps (no resonance case) for large-scale system. (b) Poincaré maps (no resonance case) for reduced system: 'linear approach'. (c) Poincaré maps (no resonance case) for reduced system: 'Direct DAE solution'. (d) Poincaré maps (no resonance case) for reduced system: 'Nonlinear projection via fixed-point iteration'. (e) Poincaré maps (no resonance case) for reduced system: 'Nonlinear (Post-processing) approach'. (f) Poincaré maps (no resonance case) for reduced system: 'Invariant Manifold approach'. 
250 S. C. Sinha et al.

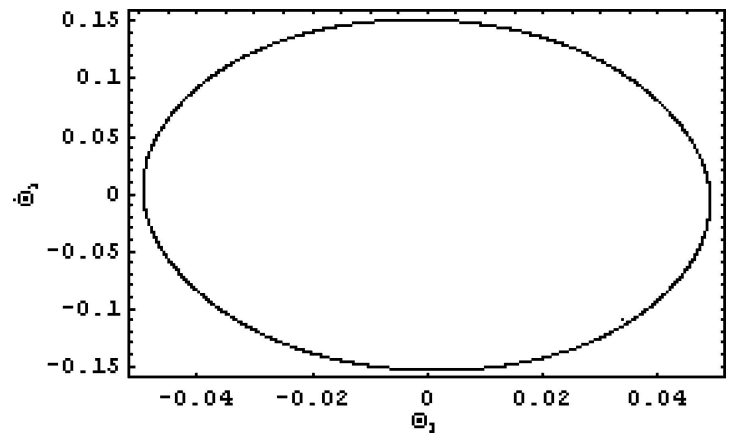

(c)

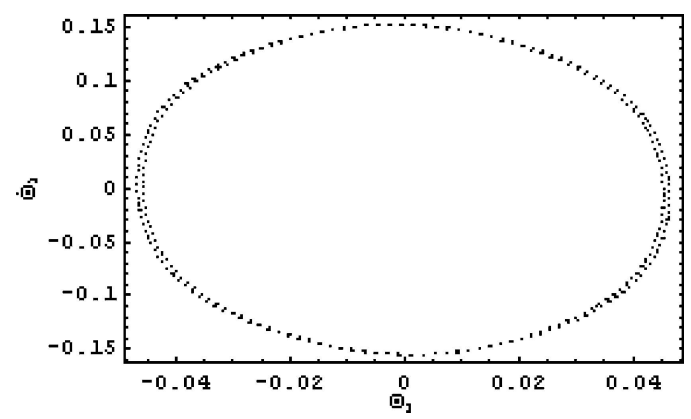

(d)

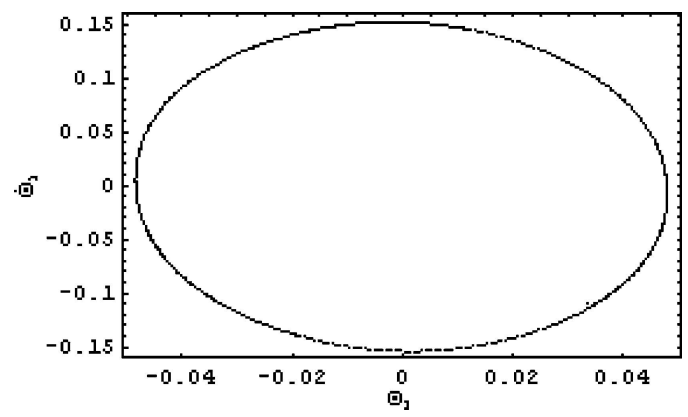

(e)

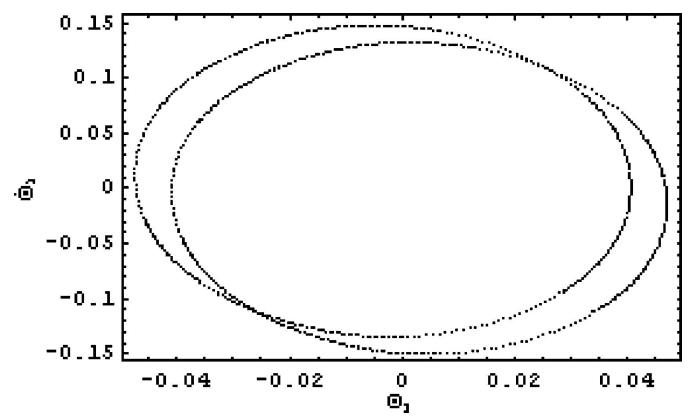

(f)

Figure 3. (Continued.) 


\subsubsection{Order Reduction Using the Nonlinear Projection via Singular Perturbation (SP) Method}

Once again, $\mathbf{z}_{r}=\left\{\begin{array}{ll}z_{1} & z_{2}\end{array}\right\}^{\mathrm{T}}$ are taken as states to be retained. By following the procedure discussed in Section 2.2, the derivatives of the non-dominant states are assumed to be small compared to the derivatives of the dominant states and Equation (31) is rewritten as

$$
\left\{\begin{array}{c}
\dot{z}_{1} \\
\dot{z}_{2} \\
\varepsilon \dot{z}_{3} \\
\varepsilon \dot{z}_{4}
\end{array}\right\}=\left[\begin{array}{cccc}
0 & -0.47 & 0 & 0 \\
0.47 & 0 & 0 & 0 \\
0 & 0 & 0 & -1.03 \\
0 & 0 & 1.03 & 0
\end{array}\right]\left\{\begin{array}{l}
z_{1} \\
z_{2} \\
z_{3} \\
z_{4}
\end{array}\right\}+\left\{\begin{array}{l}
w_{1}(\mathbf{z}, t) \\
w_{2}(\mathbf{z}, t) \\
w_{3}(\mathbf{z}, t) \\
w_{4}(\mathbf{z}, t)
\end{array}\right\}
$$

As $\varepsilon \rightarrow 0$, it yields a set of DAEs which can be integrated directly using Mathematica- $5^{\mathrm{TM}}$ and using proper transformations, all the states in $\mathbf{x}$ can be obtained. The time trace and the Poincare map are shown in Figures 2(b) and 3(c), respectively. It can be seen that the time trace of the reduced order system matches reasonably well with that of the large- scale system. However, the 'band' like structure is absent in the Poincare map of the reduced system. Alternatively, one can use single fixed-point iteration, as discussed earlier and obtain explicit expressions for $z_{3}$ and $z_{4}$ from bottom half of Equation (33) as

$$
\left\{\begin{array}{l}
z_{3} \\
z_{4}
\end{array}\right\}=-\left[\begin{array}{cc}
0 & -1.03 \\
1.03 & 0
\end{array}\right]^{-1}\left\{\begin{array}{l}
w_{3}\left(z_{1}, z_{2}, 0,0, t\right) \\
w_{4}\left(z_{1}, z_{2}, 0,0, t\right)
\end{array}\right\}
$$

Substituting $z_{3}$ and $z_{4}$ from Equation (34) into the top half of Equation (33), the reduced order model is given by

$$
\left\{\begin{array}{l}
\dot{z}_{1} \\
\dot{z}_{2}
\end{array}\right\}=\left[\begin{array}{cc}
0 & -0.47 \\
0.47 & 0
\end{array}\right]\left\{\begin{array}{l}
z_{1} \\
z_{2}
\end{array}\right\}+\left\{\begin{array}{l}
\bar{w}_{1}\left(z_{1}, z_{2}, t\right) \\
\bar{w}_{2}\left(z_{1}, z_{2}, t\right)
\end{array}\right\}
$$

Equation (35) is integrated numerically and $\mathbf{x}$ vector is constructed as before.

Once again, we compare the time trace of $\dot{\theta}_{1}$ obtained using the nonlinear projection technique with the time trace of $\dot{\theta}_{1}$ obtained by integrating the original Equation (31). It can be seen that these time traces (shown in Figure 2c) match better when compared to the time traces obtained by the linear method (Figure 2a).

It is observed that the Poincare map of reduced system (shown in Figure 3d) via nonlinear projection, is close to the Poincaré map of the large-scale system (Figure 3a) due to the fact that the reduced system also shows a narrow 'band' similar the Poincaré map of the original large-scale system implying that the dominant frequencies of the large-scale system are captured. Therefore, we conclude that the nonlinear projection (via fixed-point iteration) yields a better-reduced order model than the linear method.

\subsubsection{Order Reduction Using the Post-Processing Method}

As before, $\mathbf{z}_{r}=\left\{\begin{array}{ll}z_{1} & z_{2}\end{array}\right\}^{\mathrm{T}}$ are retained and neglecting the contribution from the non-dominant states $\mathbf{z}_{s}=\left\{\begin{array}{ll}z_{3} & z_{4}\end{array}\right\}^{\mathrm{T}}$, the system dynamics from Equation (31) is approximated by Equation (32) which can be numerically integrated to obtain the response of the system $\mathbf{z}_{r p}=\left\{\begin{array}{ll}z_{1 p} & z_{2 p}\end{array}\right\}^{\mathrm{T}}$. Then in order to obtain the post-processing correction factor $\kappa\left(z_{1}, z_{2}, t\right)$, we let $\varepsilon \rightarrow 0$ in the bottom half of Equation (33) to yield

$$
\left\{\begin{array}{l}
z_{3} \\
z_{4}
\end{array}\right\}=-\left[\begin{array}{cc}
0 & -1.03 \\
1.03 & 0
\end{array}\right]^{-1}\left\{\begin{array}{l}
\underline{w}_{3}\left(z_{1 p}(t), z_{2 p}(t), z_{3}, z_{4}, t\right) \\
\underline{w}_{4}\left(z_{1 p}(t), z_{2 p}(t), z_{3}, z_{4}, t\right)
\end{array}\right\}
$$


Since $z_{1 p}(t)$ and $z_{2 p}(t)$ are known functions of time, Equation (36) is a set of coupled nonlinear algebraic equations in $z_{3}$ and $z_{4}$ that can be solved by iterative schemes [14]. $z_{3}$ and $z_{4}$ are then substituted in $w_{1}(\mathbf{z}, t)$ and $w_{2}(\mathbf{z}, t)$ of Equation (33) to obtain $\kappa_{1}\left(z_{1}, z_{2}, t\right)$ and $\kappa_{2}\left(z_{1}, z_{2}, t\right)$, respectively. Finally, the response of the dominant modes is given as

$$
\mathbf{z}_{r}=\mathbf{z}_{r p}+\kappa\left(z_{1 p}, z_{2 p}, t\right)
$$

The time traces of the reduced order model and large-scale model are compared in Figure 2d. It can be seen that these time-traces match quite well. The long-term dynamics is represented by the Poincaré map (shown in Figure 3e). It is qualitatively similar to the Poincaré map of the reduced system using linear method (see Figure 3a). This is expected as the post-processing method tries to 'uplift' the 'flat' manifold obtained by the linear method; it may affect the magnitude of the states (thus, the time traces match very well) but not the manifold structure, significantly. Thus, the band structure present in the original large-scale system is absent in the reduced order system using the post-processing method. When the objective of order reduction is just to evaluate states for a short period, this approach is computationally faster than the nonlinear projection technique and more accurate than the linear approach. However, for some cases like 'parametric resonance' and/or 'true internal resonance' when the linear 'flat' manifold approximation does not yield correct results, it is anticipated that this post-processing approach will not yield accurate results.

\subsubsection{Order Reduction Using the Invariant Manifold Method}

As discussed in Section 2.4, in this approach the non-dominant states are expressed in terms of the dominant states by a time periodic nonlinear transformation. If the system does not exhibit 'true internal resonance' (like the case under consideration) then the 'reducibility condition' is satisfied and the system order can be reduced. We start with Equation (31) and select the same states, $\mathbf{z}_{r}=\left\{\begin{array}{ll}z_{1} & z_{2}\end{array}\right\}^{\mathrm{T}}$ as the dominant states and attempt to find a nonlinear time periodic relationship of the form given in Equation (20). The 'master/slave' relationship is given by

$$
\left(\begin{array}{ll}
z_{3} & z_{4}
\end{array}\right)^{\mathrm{T}}=\sum_{i} \mathbf{h}_{i}\left(z_{1}, z_{2}, t\right) \equiv \mathbf{H}\left(z_{1}, z_{2}, t\right)
$$

where

$$
\mathbf{h}_{i}=\sum_{\overline{\mathbf{m}}} \overline{\mathbf{h}}_{i}(t) z_{1}^{m_{1}} z_{2}^{m_{2}}, \quad \overline{\mathbf{m}}=\left(m_{1}, m_{2}\right)^{\mathrm{T}}, \quad m_{1}+m_{2}=3
$$

Here $\overline{\mathbf{h}}_{i}(t)$ are the unknown periodic vector coefficients with period $2 T$. Substituting Equation (38) into Equation (31), expanding the known and unknown periodic coefficients in Fourier series, and neglecting the terms of higher order, we obtain the relationship (for more details, see [14])

$$
\begin{aligned}
z_{3}= & z_{1} z_{2}^{2}(0.036-0.002 \cos (2 \pi t))+z_{1}^{3}(0.038-0.004 \cos (2 \pi t)+0.001 \cos (4 \pi t)) \\
& +z_{2}^{3}(-0.001 \sin (2 \pi t))+z_{1}^{2} z_{2}(-0.002 \sin (2 \pi t)+0.001 \sin (4 \pi t))=H_{3}\left(z_{1}, z_{2}, t\right) \\
z_{4}= & z_{1} z_{2}^{2}(0.074-0.021 \cos (2 \pi t)-0.005 \cos (4 \pi t))+z_{2}^{3}(0.061) \\
& +z_{1}^{3}(-0.017 \sin (2 \pi t)-0.003 \sin (4 \pi t))+z_{2} z_{1}^{2}(-0.005 \sin (2 \pi t)-0.002 \sin (4 \pi t)) \\
= & H_{4}\left(z_{1}, z_{2}, t\right)
\end{aligned}
$$


Equation (39) is substituted into the top half of Equation (31) to obtain the reduced order model as

$$
\left\{\begin{array}{l}
\dot{z}_{1} \\
\dot{z}_{2}
\end{array}\right\}=\left[\begin{array}{cc}
0 & -0.47 \\
0.47 & 0
\end{array}\right]\left\{\begin{array}{l}
z_{1} \\
z_{2}
\end{array}\right\}+\left\{\begin{array}{l}
\underline{w}_{1}\left(z_{1}, z_{2}, H_{3}\left(z_{1}, z_{2}, t\right), H_{4}\left(z_{1}, z_{2}, t\right), t\right) \\
\underline{w}_{2}\left(z_{1}, z_{2}, H_{3}\left(z_{1}, z_{2}, t\right), H_{4}\left(z_{1}, z_{2}, t\right), t\right)
\end{array}\right\}
$$

This symbolic computation was carried out using Mathematica ${ }^{\text {TM }}$. Equation (40) is integrated numerically to determine response of the reduced order system.

Once again the time trace of $\dot{\theta}_{1}$ obtained by this method is compared with the time trace of $\dot{\theta}_{1}$ (Figure 2e) obtained via numerical integration of the original system given by Equation (31). As we can see, the difference between the two time traces is hardly distinguishable. Even the Poincare map of the original system (Figure 3a) matches the map of the reduced order system (Figure 3f) quantitatively. The map of the reduced system has 2 loops which is an approximation of the 'band' present in the Poincare map of the original system. Therefore, it may be concluded that the invariant manifold order reduction technique yields the most accurate reduced order model when compared to the 'linear' and the 'nonlinear projection' methods.

In order to compare all order reduction procedures, we carry out numerical error analysis. Here we define the error norm as the absolute value of the difference between the time traces of the large-scale system and the reduced order system. This error is plotted as a function of initial conditions for various order reduction approaches for the case of no resonance in Figure 4. It can be seen that for small initial conditions the error is not so significant, however, as the initial conditions become large, the error due to linear approximation increases drastically. Amongst the nonlinear methodologies the error due to the invariant manifold approach is least followed by the singular perturbation approach and post-processing method.

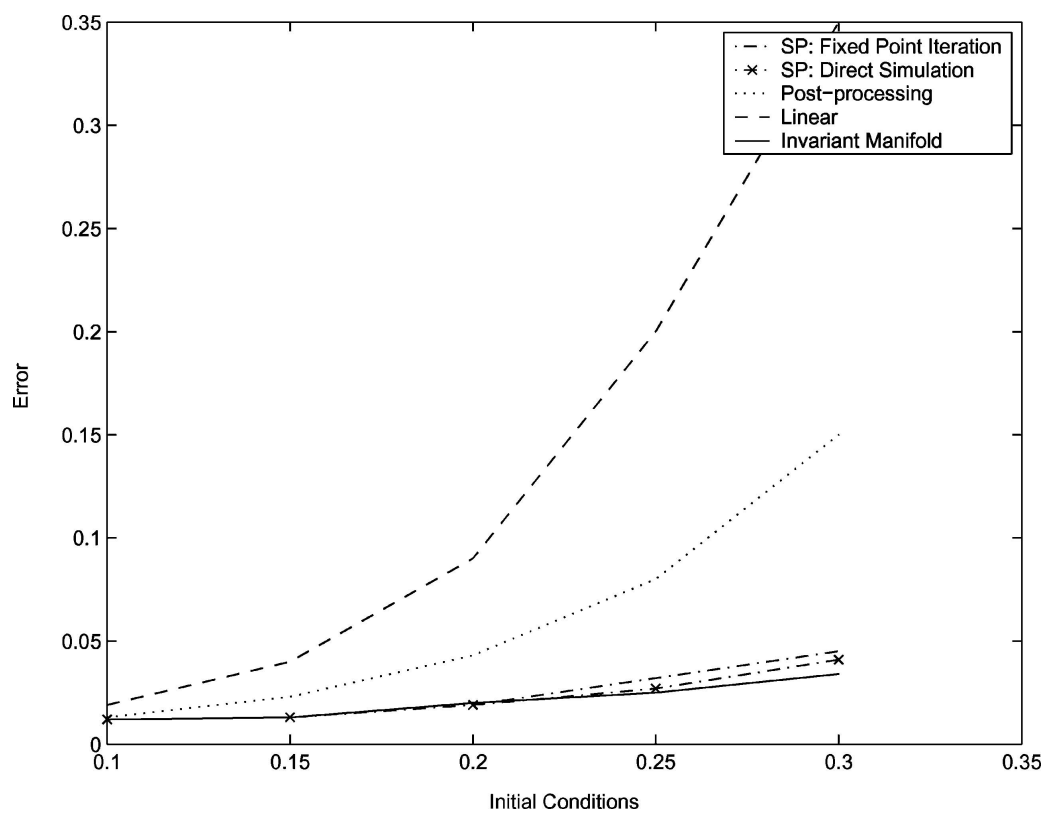

Figure 4. Comparison of error (no resonance case). 
Table 2. Parameter values for the case when 'true internal resonance' exists.

\begin{tabular}{lllllllll}
\hline Parameter & $\omega_{n_{1}}^{2}$ & $\omega_{n_{2}}^{2}$ & $\varepsilon p_{1}$ & $\varepsilon p_{2}$ & $\omega$ & $b$ & $c$ & $d$ \\
\hline Value & 4.5 & 4 & 0.1 & 4.5 & $2 \pi$ & 3.5 & 0 & 1 \\
\hline
\end{tabular}

\subsection{TRUe InTERnal ResonanCE CASE}

For time periodic systems, a 'true internal resonance' is said to occur when some linear combination of the $\lambda_{l}$ (Floquet exponents of the dominant states) and $\bar{\lambda}_{p}$ (one of the Floquet exponents of the non-dominant states) add up to zero for $v=0$ (cf., Equation (27)). For illustration, we select the system parameters as given in Table 2. For this set of parameters, the Floquet multipliers are $(-0.96 \pm$ $0.26 \bar{i}, 0.57 \pm 0.81 \bar{i})$ and the Floquet exponents are $( \pm 0.95 \bar{i}, \pm 2.86 \bar{i})$. In this case, the 'reducibility condition' is not satisfied since for $v=0$, Equation (27) yields $3 \times 0.95 \bar{i}-2.86 \bar{i}=0$. Note that $m_{1}=3, m_{2}=0 ; \lambda_{1}, \lambda_{2}= \pm 0.95 \bar{i} \& \bar{\lambda}_{1}, \bar{\lambda}_{2}= \pm 2.86 \bar{i}$. Thus, the modes cannot be decoupled and order reduction is not possible using the invariant manifold approach. However, linear or nonlinear projection based order reduction is certainly possible and they may yield acceptable results for very small initial conditions when the nonlinear effects are not significant.

\subsection{Parametric Resonance Case}

As discussed in Section 2.3, in the case of 'parametric resonance' at least one pair of Floquet multipliers is repeated. This implies that the eigenvalues of $\mathbf{J}$ matrix corresponding to the resonant modes are zero. It can be observed from the 'reducibility condition' (cf., Equation (27)), for an accurate order reduction via invariant manifold approach the resonant modes should be selected as the 'master' coordinates to be retained and the reduced order model is purely nonlinear. It is intersecting to note that order reduction using singular perturbation via fixed-point iteration approach also imposes that the resonant modes must be chosen as the 'master' states. However, the linear approach does not yield such kind of condition and fails to obtain meaningful results. The details can be found in references [14, 21, 23].

\section{Direct Order Reduction of a Set of Second Order Equations}

\subsection{THE INVARIANT MANIFOLD APPROACH}

In the following, we also attempt to construct a direct methodology of order reduction for a set of second order equations with time periodic coefficients, which naturally arise in structural modeling [15]. Burton et al. [7, 8] have proposed such techniques for a set of second order autonomous systems.

Consider the $m$ dimensional system in structural (second order) form given by

$$
\mathbf{M}(t) \ddot{\mathbf{y}}+\mathbf{K}(t) \mathbf{y}+\mathbf{F}(\mathbf{y}, \dot{\mathbf{y}}, t)=\mathbf{0}
$$

which can be written as

$$
\ddot{\mathbf{y}}+\hat{\mathbf{K}}(t) \mathbf{y}+\hat{\mathbf{f}}(\mathbf{y}, \dot{\mathbf{y}}, t)=\mathbf{0}
$$


where $\hat{\mathbf{K}}(t)=\mathbf{M}^{-1}(t) \mathbf{K}(t)$ and $\hat{\mathbf{f}}()=\mathbf{M}^{-1}(t) \mathbf{F}()$. It is desired to obtain a reduced order model of the form

$$
\ddot{\tilde{\mathbf{y}}}_{r}+\tilde{\mathbf{K}}_{r} \tilde{\mathbf{y}}_{r}+\mathbf{F}\left(\tilde{\mathbf{y}}_{r}, \dot{\tilde{\mathbf{y}}}_{r}, t\right)=0, \quad r \ll m
$$

It is to be noted that matrix $\tilde{\mathbf{K}}_{r}$ is a time invariant matrix. Therefore, we have a slightly different problem than before. The system described by Equation (42) can be expressed as a set of $n=2 \times m$ first order equations given by Equation (4) where $\mathbf{x}=(\mathbf{y}, \dot{\mathbf{y}})^{\mathrm{T}}$. Then, applying the L-F transformation $\mathbf{x}=\mathbf{Q}(t) \overline{\mathbf{y}}$ yields

$$
\dot{\overline{\mathbf{y}}}(t)=\mathbf{R} \overline{\mathbf{y}}(t)+\mathbf{Q}^{-1}(t) \mathbf{f}(\overline{\mathbf{y}}, t)
$$

Using another transformation,

$$
\overline{\mathbf{y}}(t)=\left[\begin{array}{cc}
-\mathbf{R}_{11}^{-1} \mathbf{R}_{12} & \mathbf{0} \\
\mathbf{I} & \mathbf{I}
\end{array}\right]\left[\begin{array}{l}
\mathbf{v}_{1} \\
\mathbf{v}_{2}
\end{array}\right] \equiv \mathbf{G v}(t), \quad \mathbf{R}=\left[\begin{array}{ll}
\mathbf{R}_{11} & \mathbf{R}_{12} \\
\mathbf{R}_{21} & \mathbf{R}_{22}
\end{array}\right]
$$

we obtain

$$
\left[\begin{array}{c}
\dot{\mathbf{v}}_{1} \\
\dot{\mathbf{v}}_{2}
\end{array}\right]=\left[\begin{array}{cc}
\mathbf{0} & \overline{\mathbf{M}}^{-1} \\
-\overline{\mathbf{K}}^{-1} & \mathbf{0}
\end{array}\right]\left[\begin{array}{l}
\mathbf{v}_{1} \\
\mathbf{v}_{2}
\end{array}\right]+\mathbf{G}^{-1} \mathbf{Q}^{-1} \mathbf{f}(\mathbf{Q G v}, t)
$$

where $\overline{\mathbf{M}}^{-1}=-\mathbf{R}_{12}^{-1} \mathbf{R}_{11}^{-1} \mathbf{R}_{12}$ and $\overline{\mathbf{K}}^{-1}=-\mathbf{R}_{21}^{-1} \mathbf{R}_{11}^{-1} \mathbf{R}_{12}$. If $\mathbf{R}_{11}$ is not invertible, then a similar transformation exists using $\mathbf{R}_{22}^{-1}$ [29]. Differentiating the upper partition, pre-multiplying the lower partition by $\overline{\mathbf{M}}^{-1}$ and equating yields

$$
\ddot{\mathbf{v}}_{1}+\overline{\mathbf{M}}^{-1} \overline{\mathbf{K}} \mathbf{v}_{1}-\overline{\mathbf{M}}^{-1} \tilde{\mathbf{f}}_{2}\left(\mathbf{v}_{1}, \mathbf{v}_{2}, t\right)-\frac{\partial \tilde{\mathbf{f}}_{1}}{\partial \mathbf{v}_{1}} \dot{\mathbf{v}}_{1}-\frac{\partial \tilde{\mathbf{f}}_{1}}{\partial \mathbf{v}_{2}}\left(-\overline{\mathbf{K}} \mathbf{v}_{1}+\hat{\mathbf{f}}_{2}\right)-\frac{\partial \tilde{\mathbf{f}}_{1}}{\partial t}=0
$$

where the order of nonlinearity is kept the same as in Equation (41). Setting $\tilde{\mathbf{y}}=\mathbf{v}_{1}$ this Equation may be written as

$$
\ddot{\tilde{\mathbf{y}}}+\tilde{\mathbf{K}} \tilde{\mathbf{y}}+\tilde{\mathbf{f}}(\tilde{\mathbf{y}}, \dot{\tilde{\mathbf{y}}}, t)=0
$$

This Equation is a time invariant form of nonlinear time periodic system given by Equation (41). The $m$ dimensional displacement vector $\tilde{\mathbf{y}}$ is assumed to have $r$ 'master' degrees of freedom $\tilde{\mathbf{y}}_{r}$ and $s$ 'slave' degrees of freedom $\tilde{\mathbf{y}}_{s}$. Equation (48) is partitioned as

$$
\left[\begin{array}{c}
\ddot{\mathbf{y}}_{s} \\
\ddot{\mathbf{y}}_{r}
\end{array}\right]+\left[\begin{array}{cc}
\tilde{\mathbf{K}}_{11} & \tilde{\mathbf{K}}_{12} \\
\tilde{\mathbf{K}}_{21} & \tilde{\mathbf{K}}_{22}
\end{array}\right]\left[\begin{array}{c}
\tilde{\mathbf{y}}_{s} \\
\tilde{\mathbf{y}}_{r}
\end{array}\right]+\left[\begin{array}{l}
\tilde{\mathbf{f}}_{1}\left(\tilde{\mathbf{y}}_{r}, \tilde{\mathbf{y}}_{s}, \dot{\tilde{\mathbf{y}}}_{r}, \dot{\tilde{\mathbf{y}}}_{s}, t\right) \\
\tilde{\mathbf{f}}_{2}\left(\tilde{\mathbf{y}}_{r}, \tilde{\mathbf{y}}_{s}, \dot{\mathbf{y}}_{r}, \dot{\mathbf{y}}_{s}, t\right)
\end{array}\right]=0
$$

The 'slave' degrees of freedom are to be eliminated by the 'master-slave' relationship. Similar to Burton and Rhee [7] approach for autonomous system, we express $\tilde{\mathbf{y}}_{s}$ as

$$
\tilde{\mathbf{y}}_{s}=\overline{\mathbf{T}} \tilde{\mathbf{y}}_{r}+\widehat{\mathbf{g}}\left(\tilde{\mathbf{y}}_{r}, \dot{\tilde{\mathbf{y}}}_{r}, t\right)
$$


such that $\mathbf{g}$ has the same degree of nonlinearity and periodicity in time as $\tilde{\mathbf{f}}_{i}$ in Equation (49) and $\widehat{\mathbf{g}}(0,0, t)=0$. The nonlinear transformation term in Equation (50) is expressed as

$$
\widehat{\mathbf{g}}\left(\tilde{\mathbf{y}}_{r}, \dot{\tilde{\mathbf{y}}}_{r}, t\right)=\sum_{(i, \tilde{n})} g_{i, \tilde{n}}(t) \tilde{y}_{m_{1}}^{n_{1}} \ldots \tilde{y}_{m_{m}}^{n_{m}} \dot{\tilde{y}}_{m_{1}}^{n_{m+1}} \ldots \tilde{y}_{m_{m}}^{n_{2 m}} e_{i}
$$

where,

$$
\tilde{n}=\left(n_{1}, \ldots, n_{m}, n_{m+1}, \ldots, n_{2 m}\right) ; \quad \sum_{k} n_{k}=l
$$

$l$ is the order of the nonlinear terms retained in $\tilde{\mathbf{f}}_{i}$ and each $g_{i, \tilde{n}}(t)$ is a Fourier series with unknown coefficients. Equations (49) and (50) yield conditions that matrix $\overline{\mathbf{T}}$ and vector $\mathbf{g}$ must satisfy. These are

$$
\begin{aligned}
& \overline{\mathbf{T}}=-\left(\tilde{\mathbf{K}}_{11}-\overline{\mathbf{T}} \tilde{\mathbf{K}}_{21}\right)^{-1}\left(\tilde{\mathbf{K}}_{12}-\overline{\mathbf{T}} \tilde{\mathbf{K}}_{22}\right) \\
& \ddot{\mathbf{g}}+\left(\tilde{\mathbf{K}}_{11}-\overline{\mathbf{T}} \tilde{\mathbf{K}}_{21}\right) \widehat{\mathbf{g}}=\overline{\mathbf{T}}_{2}-\tilde{\mathbf{f}}_{1}
\end{aligned}
$$

Equation (52) is first solved by iteration for $\overline{\mathbf{T}}$ using MATLAB ${ }^{\mathbf{T M}}$ and then Equation (53) is solved for the Fourier coefficients of $\hat{\mathbf{g}}$ from Equations (51) and (53) via harmonic balance. As discussed in [15], the matrix $\overline{\mathbf{T}}$ is an extension of the Guyan reduction matrix [2], which accounts for both stiffness and inertia effects and thus allows the reduced model to preserve the eigen-structure projected onto the $\mathbf{y}_{r}$ coordinates.

\subsection{THE LiNEAR ORDER REDUCTION APPROACH}

If a linear order reduction transformation is applied such that $\mathbf{g}() \equiv 0$ in Equation (50), then a nonlinear reduced order model is given by

$$
\ddot{\tilde{\mathbf{y}}}_{r}+\tilde{\mathbf{K}}_{22} \tilde{\mathbf{y}}_{r}+\tilde{\mathbf{f}}\left(\tilde{\mathbf{y}}_{r}, \dot{\tilde{\mathbf{y}}}_{r}, t\right)=0
$$

while the slave states are given only by the linear part of Equation (50). Results from this linear-based order reduction procedure will be compared with those obtained from the nonlinear reduced order model of Equation (41) in Section 5.

\subsection{Derivation of Reducibility (Resonance) Conditions}

One can also obtain various 'reducibility (resonance) conditions' derived in Section 2 via direct second order formulation as follows. Consider the set of equations given by Equation (49), using the modal transformation $\tilde{\mathbf{y}}=\overline{\mathbf{M}} \overline{\mathbf{y}}$, we obtain

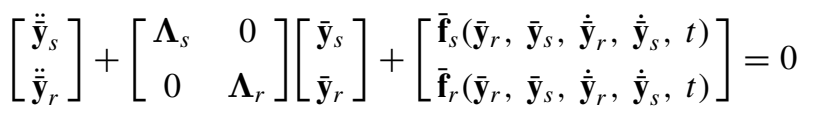

where $\boldsymbol{\Lambda}_{s}, \boldsymbol{\Lambda}_{r}$ are diagonal matrices assuming that $\tilde{\mathbf{K}}$ in Equation (48) or (49) is symmetric (or symmetrizable) and positive definite. The entries $\boldsymbol{\Lambda}_{s}$ and $\boldsymbol{\Lambda}_{r}$ are squares of the Floquet exponents corresponding to the non-dominant modes $\left(\boldsymbol{\lambda}_{s}^{2}\right)$ and dominant modes $\left(\boldsymbol{\lambda}_{r}^{2}\right)$, respectively. As the linear part is uncoupled, using a purely nonlinear master-slave relationship given by

$$
\left.\overline{\mathbf{y}}_{s}=\widehat{\mathbf{g}}_{\left(\overline{\mathbf{y}}_{r}\right.}, \dot{\overline{\mathbf{y}}}_{r}, t\right)
$$


$\ddot{\overline{\mathbf{y}}}_{s}$ is computed using (56) as

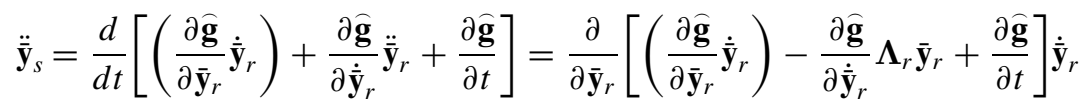

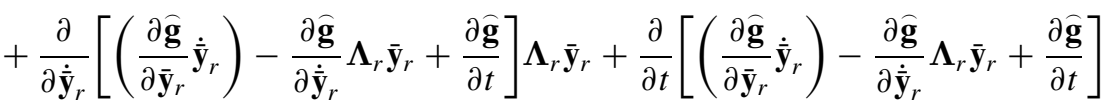

Neglecting higher order terms and equating Equations (57) and (55) yields a complex partial differential Equation in $\mathbf{g}$. Assuming that each of the $g_{i, \tilde{n}}(t)$ has a Fourier series representation given by

$$
g_{i, \tilde{n}}(t)=\sum_{\nu} g_{i, \tilde{n}, \nu} e^{\bar{i} \nu \Omega t} ; \quad \Omega=\frac{\pi}{T}
$$

We substitute Equation (58) in (57) and (55) and equate terms to obtains a set of complex linear algebraic equations for the unknown Fourier coefficients in Equation (58). Let the set of complex equations be represented by

$$
\tilde{\mathbf{D}}(v) \tilde{\mathbf{g}}_{v}=\tilde{\mathbf{k}}_{v}
$$

where $\tilde{\mathbf{g}}_{v}$ is a vector of unknown Fourier coefficients in Equation (58) and $\tilde{\mathbf{k}}_{v}$ is the corresponding vector of known coefficients. From Equation (59) it is immediately observed that $\tilde{\mathbf{D}}(v)$ must be invertible and the 'reducibility condition' requires that $\operatorname{det}[\tilde{\mathbf{D}}(v)] \neq 0$. If $\operatorname{det}[\tilde{\mathbf{D}}(v)]=0$ then various resonances may occur and nonlinear order reduction is not possible. At this point, it is rather instructive to consider various special forms of nonlinearities and determine the corresponding 'reducibility (resonance) conditions'.

First, consider reducing the system given by Equation (55) to a single degree of freedom reduced model with quadratic nonlinearities in $\mathbf{g}$. The $3 \times 3$ complex coefficient matrix in Equation (59) can be computed in terms of the 'master exponent' $\left(\lambda_{1}\right)$ and any of the 'slave exponents' $\left(\lambda_{s}\right)$ as

$$
\tilde{\mathbf{D}}(v)=\left[\begin{array}{ccc}
\lambda_{s}^{2}-2 \lambda_{1}^{2}-v^{2} \Omega^{2} & -2 \bar{i} v \Omega \lambda_{1}^{2} & 2 \lambda_{1}^{4} \\
4 \bar{i} \nu \Omega & \lambda_{s}^{2}-4 \lambda_{1}^{2}-v^{2} \Omega^{2} & -4 \bar{i} v \Omega \lambda_{1}^{2} \\
2 & 2 \bar{i} v \Omega & \lambda_{s}^{2}-2 \lambda_{1}^{2}-v^{2} \Omega^{2}
\end{array}\right]
$$

For $v=0$, the 2:1 'true internal resonance' condition $\lambda_{s}=2 \lambda_{1}$ is obtained by solving for $\lambda_{s}$ from the Equation $\operatorname{det}[\tilde{\mathbf{D}}(0)]=0$. For $v \neq 0$, the 'true combination resonance' conditions are obtained from the Equation $\operatorname{det}[\tilde{\mathbf{D}}(v)]=0$ as $\lambda_{s}=\left|2 \lambda_{1} \pm v \Omega\right|$ and $\lambda_{s}=v \Omega$.

For a single degree-of-freedom reduced model with cubic nonlinearities in $\widehat{\mathbf{g}}$ the complex $4 \times 4$ coefficient matrix is given as

$$
\tilde{\mathbf{D}}(v)=\left[\begin{array}{cccc}
\lambda_{s}^{2}-3 \lambda_{1}^{2}-v^{2} \Omega^{2} & -2 \bar{i} v \Omega \lambda_{1}^{2} & 2 \lambda_{1}^{4} & 0 \\
6 \bar{i} v \Omega & \lambda_{s}^{2}-7 \lambda_{1}^{2}-v^{2} \Omega^{2} & -4 \bar{i} v \Omega \lambda_{1}^{2} & 2 \lambda_{1}^{4} \\
6 & 4 \bar{i} v \Omega & \lambda_{s}^{2}-7 \lambda_{1}^{2}-v^{2} \Omega^{2} & -6 \bar{i} v \Omega \lambda_{1}^{2} \\
0 & 2 & 2 \bar{i} v \Omega & \lambda_{s}^{2}-3 \lambda_{1}^{2}-v^{2} \Omega^{2}
\end{array}\right]
$$

Substituting $v=0$ in Equation (61) and setting $\operatorname{det}[\tilde{\mathbf{D}}(v)]=0$ gives 3:1 and 1:1 'true internal resonance' conditions $\lambda_{s}=3 \lambda_{1}, \lambda_{s}=\lambda_{1}$. The 'true combination resonance' conditions for $v \neq 0$ are obtained as $\lambda_{s}=\left|\lambda_{1} \pm v \Omega\right|,\left|3 \lambda_{1} \pm v \Omega\right|$. It can be observed that the conditions presented here are the same 
conditions obtained via 'reducibility condition' given by Equation (27) in Section 2.4. Also note that when the parametric excitation is small then $\lambda_{1} \rightarrow \omega_{n_{1}}$, the natural frequency of linearized autonomous system corresponding to the 'master' state and the results for 'internal resonance' are recovered as shown in Section 2.4 .

\section{An Application}

Consider a double inverted pendulum as shown in Figure 5. The local equations of motion for a double inverted pendulum with periodic semi-follower force expanded up to cubic terms about $\left(x_{1}, x_{2}\right)=(0,0)$ are given by

$$
\begin{aligned}
& {\left[\begin{array}{l}
\ddot{x}_{1} \\
\ddot{x}_{2}
\end{array}\right]+\left[\begin{array}{cc}
0.5 \bar{k}(3-\bar{p}) & 0.5 \bar{k}(\bar{p}-2) \\
0.5 \bar{k}(\bar{p}-5) & \bar{k}(2-\bar{p}(1.5-\gamma))
\end{array}\right]\left[\begin{array}{l}
x_{1} \\
x_{2}
\end{array}\right]} \\
& +\left[\begin{array}{c}
0.5\left(\dot{x}_{1}^{2}+\dot{x}_{2}^{2}\right)\left(x_{1}-x_{2}\right)-\bar{p} \bar{k}\left[\left(x_{1}-\gamma x_{2}\right)^{3}-(1-\gamma)^{3} x_{2}^{3}\right] / 12 \\
+0.25\left(x_{1}-x_{2}\right)^{2}\left[\bar{k}(\bar{p}-4) x_{1}+\bar{k}(3+\bar{p}(\gamma-2)) x_{2}\right] \\
-0.5\left(3 \dot{x}_{1}^{2}+\dot{x}_{2}^{2}\right)\left(x_{1}-x_{2}\right)-\bar{p} \bar{k}\left[\left(x_{1}-\gamma x_{2}\right)^{3}-3(1-\gamma)^{3} x_{2}^{3}\right] / 12 \\
-0.25\left(x_{1}-x_{2}\right)^{2}\left[\bar{k}(2 \bar{p}-7) x_{1}+\bar{k}(5+\bar{p}(\gamma-3)) x_{2}\right]
\end{array}\right]=0
\end{aligned}
$$

where $\bar{p}=\bar{p}_{1}+\bar{p}_{2} \cos (\Omega t)$ is the source of time periodic stiffness matrix and periodically modulated nonlinearities. For a particular parameter set $\bar{p}_{1}=1, \bar{p}_{2}=0.7, \bar{k}=1.2, \gamma=1, \Omega=2$, it is found

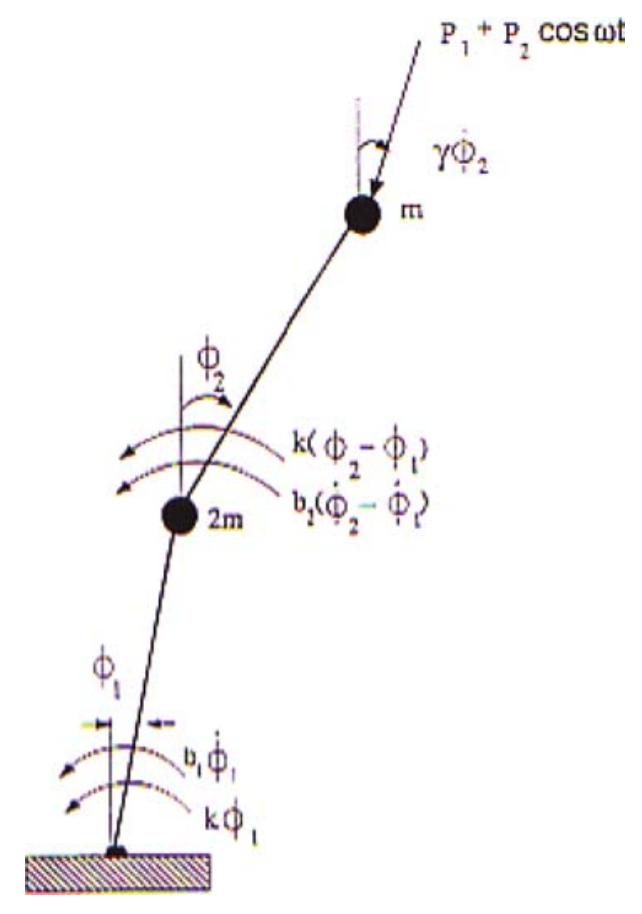

Figure 5. Double inverted pendulum with $b_{1}=b_{2}=0$. 
that the transformed nonlinear system in Equation (49) is given by

$$
\left[\begin{array}{l}
\ddot{y}_{1} \\
\ddot{y}_{2}
\end{array}\right]+\left[\begin{array}{ll}
0.9855 & 0.7846 \\
0.4670 & 2.1343
\end{array}\right]\left[\begin{array}{l}
y_{1} \\
y_{1}
\end{array}\right]+\left[\begin{array}{l}
\bar{f}_{1}\left(y_{1}, \dot{y}_{1}, y_{2}, \dot{y}_{2}, t\right) \\
\bar{f}_{2}\left(y_{1}, \dot{y}_{1}, y_{2}, \dot{y}_{2}, t\right)
\end{array}\right]=0
$$

where $\bar{f}_{1}$ and $\bar{f}_{2}$ contain periodically modulated cubic nonlinearities. The normalized eigenvalues of the time invariant system matrix $\mathbf{R}$ (Floquet exponents corresponding to original time periodic system) are $\pm 0.8517 \bar{i}, \pm 1.5474 \bar{i}$ whereas the eigenvalues of the constant matrix in Equation (49) are: $\lambda_{s}^{2}=2.12$ and $\lambda_{r}^{2}=0.74$. These are in agreement. The ratio $\frac{\lambda_{s}}{\lambda_{r}}$ is 1.8168 , which is not in the vicinity of $1: 1$ or $3: 1$ 'true internal resonances'. Because the process of computing and applying the $2 T$-periodic L-F transformation requires normalization of the principal period to unity [12], the nonlinearities in the transformed domain are periodic with frequency $\Omega=\pi$. Thus, it can be verified that the system is also not in 'true combination resonance', i.e.,

$$
1.5474 \neq|0.8517 \pm v \pi|, \quad|3(0.8517) \pm v \pi|
$$

The reduced order model using the transformation $y_{1}=\bar{T} y_{2}+\hat{g}\left(y_{2}, \dot{y}_{2}, t\right)$ is thus obtained as

$$
\ddot{y}_{2}+\lambda_{r}^{2} y_{2}+\hat{f}\left(y_{2}, \dot{y}_{2}, t\right)=0
$$

where $\hat{f}\left(y_{2}, \dot{y}_{2}, t\right)$ contains periodically modulated cubic terms in $y_{2}$ and $\dot{y}_{2}$. Figure 6 shows the comparison between the angular displacements $x_{1}(t), x_{2}(t)$ obtained via numerical integration of the original large-scale system and from the nonlinear-based and linear-based reduced order models, respectively. It can be clearly seen that the nonlinear-based reduced order model produces a more accurate response
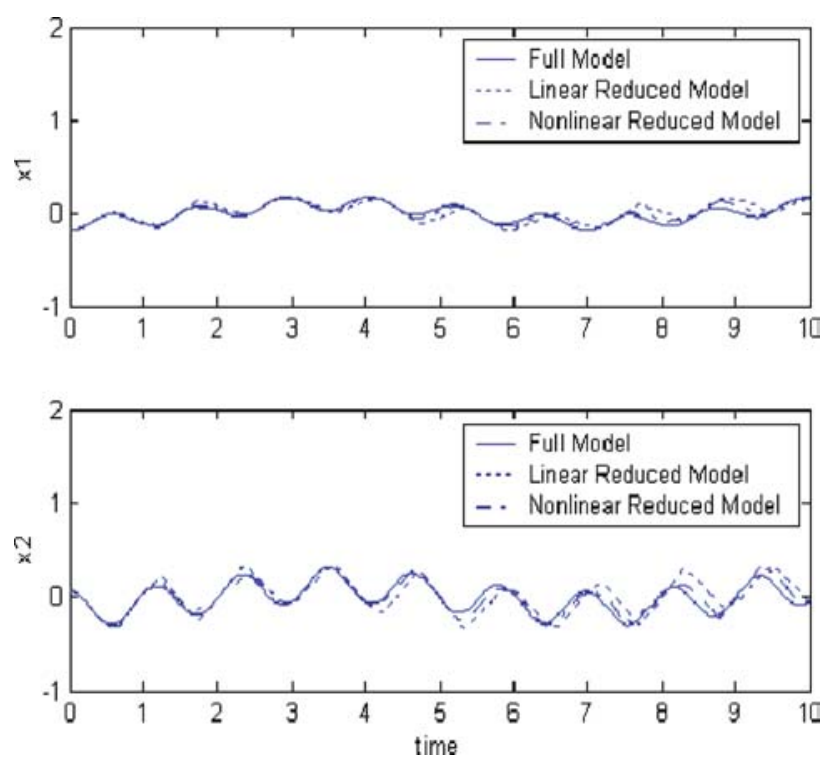

Figure 6. Comparison of the actual, nonlinear reduced and linear reduced model trajectories for double inverted pendulum away from resonance. 


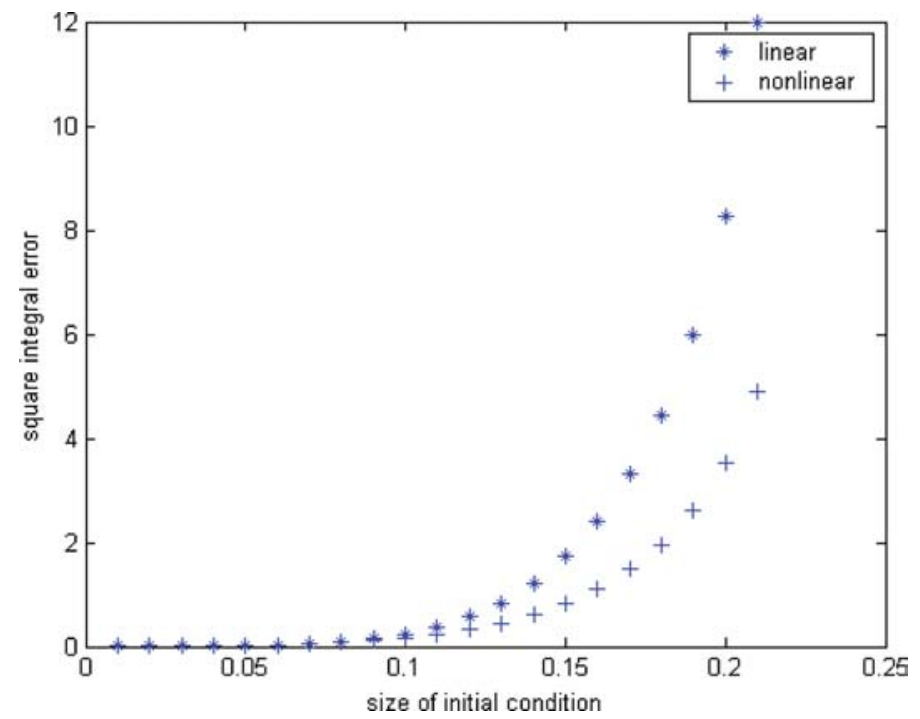

Figure 7. Integral square error for gradually increasing initial conditions for double inverted pendulum away from resonance.

than its linear counterpart and is also confirmed by Figure 7, which compares the error norm

$$
\int_{0}^{t_{f}} e^{2}(t) d t=\left[\sum_{i=1}^{N} e^{2}\left(t_{i}\right)\right] \Delta t \approx \sum_{i=1}^{N} e^{2}\left(t_{i}\right)
$$

for the responses of linear and nonlinear order reduced models with $e(t)$ being the error between the actual trajectory of the original system and the corresponding trajectories reconstructed from the two reduced models. It can be seen from Figure 7 that the nonlinear reduced order model is more accurate in terms of measure (66) than its linear counterpart as the size of the initial condition is increased.

\section{Generalization to Periodic-Quasiperiodic Systems}

Analysis of quasiperiodic system is a very active area of research. Unlike the time-periodic systems, the rigorous theories for stability do not exist as of yet for these class of problems. Here, we briefly present the order reduction schemes for a special class of quasiperiodic systems known as periodicquasiperiodic systems. These systems have periodic linear part and the nonlinearities are quasiperiodic. Very recently, a novel analysis technique based on L-F transformation and quasiperiodic normal forms was presented by Wooden [17] to study the dynamics of these types of problems.

Consider the system given by

$$
\dot{\mathbf{x}}(t)=\mathbf{A}(t) \mathbf{x}(t)+\hat{\mathbf{f}}(\mathbf{x}, t)
$$

where $\mathbf{x}$ is an $n$ vector of states, $\mathbf{A}(t)$ is an $n \times n$ periodic matrix with frequency $\omega_{1}$, as defined before $\hat{\mathbf{f}}(\mathbf{x}, t)$ is a nonlinear periodic $n$ vector with frequency $\omega_{2}$ such that $\hat{\mathbf{f}}(\mathbf{0}, t)=\mathbf{0}$ and it contains monomials of vector $\mathbf{x}$. 
Applying the L-F and modal transformations $\mathbf{x}(t)=\mathbf{Q}(t) \mathbf{M z}(t)$ produces

$$
\dot{\mathbf{z}}(t)=\mathbf{J z}(t)+\mathbf{M}^{-1} \mathbf{Q}^{-1}(t) \hat{\mathbf{f}}(\mathbf{z}, t) \equiv \mathbf{J z}(t)+\hat{\mathbf{w}}(\mathbf{z}, t)
$$

where $\mathbf{J}$ is the Jordan form of $\mathbf{R}$ and $\hat{\mathbf{w}}(\mathbf{z}, t)$ represents an appropriately defined nonlinear quasiperiodic vector consisting of monomials of $z_{j}$ whose time-periodic coefficients contain frequencies $\omega_{1}$ and $\omega_{2}$.

Again, the objective of order reduction is to replace the nonlinear time periodic system given by Equation (68) by an equivalent system given by

$$
\dot{\mathbf{z}}_{r}(t)=\mathbf{J}_{r} \mathbf{z}_{r}(t)+\hat{\mathbf{w}}_{r}\left(\mathbf{z}_{r}, t\right)
$$

One can apply the linear approach, nonlinear projection techniques or post-processing method to obtain the reduced order model described by Equation (69) in a straightforward manner. However, in order to apply the invariant manifold approach a nonlinear quasiperiodic relationship must be assumed between the non-dominant and the dominant states.

Consider the nonlinear quasiperiodic 'slave' and 'master' relation

$$
\mathbf{z}_{s}=\sum_{i} \mathbf{h}_{i}\left(\mathbf{z}_{r}, t\right) \equiv \mathbf{H}\left(\mathbf{z}_{r}, t\right)
$$

where

$$
\mathbf{h}_{i}=\sum_{\overline{\mathbf{m}}} \overline{\mathbf{h}}_{i}(t) \mathbf{z}_{1}^{m_{1}} \ldots \mathbf{z}_{r}^{m_{r}}, \quad \overline{\mathbf{m}}=\left(m_{1}, \ldots, m_{r}\right)^{\mathrm{T}}, \quad m_{1}+\cdots+m_{r}=i, \quad i=2,3, \ldots, k
$$

Here $\overline{\mathbf{h}}_{i}(t)$ are the unknown quasiperiodic vector coefficients. Separation of 'master' and 'slave' states results in an Equation similar to (8) with quasiperiodic coefficients and using Equation (70), we obtain a nonlinear partial differential Equation similar to (21). To solve this partial differential Equation approximately, we expand the known and the unknown quasiperiodic coefficient functions $\left(\overline{\mathbf{h}}_{i}(t)\right)$ into multiple Fourier series as

$$
\begin{aligned}
\overline{\mathbf{h}}_{i}\left(\mathbf{z}_{r}, t\right) & =\sum_{j=1}^{s} \sum_{\overline{\mathbf{m}}} \sum_{p_{1}=-\infty}^{+\infty} \sum_{p_{2}=-\infty}^{+\infty} h_{j \bar{m} \nu} e^{\bar{i}(\overline{\mathbf{p}} \cdot \omega) t}\left|\mathbf{z}_{r}\right|^{\mathbf{m}} e_{j} \\
\hat{\mathbf{w}}_{s i}\left(\mathbf{z}_{r}, t\right) & =\sum_{j=1}^{s} \sum_{\overline{\mathbf{m}}} \sum_{p_{1}=-\infty} \sum_{p_{2}=-\infty}^{+\infty} a_{j \bar{m} \nu} e^{\bar{i}(\overline{\mathbf{p}} \cdot \omega) t}\left|\mathbf{z}_{r}\right|^{\mathbf{m}} e_{j}
\end{aligned}
$$

where $\left|\mathbf{z}_{r}\right|^{\mathbf{m}}=z_{1}^{m_{1}} z_{2}^{m_{2}} \cdots z_{r}^{m_{r}}, \bar{i}=\sqrt{-1}, m_{1}+\cdots+m_{r}=i ; i=2,3, \ldots, k, \boldsymbol{\omega}=\left\{\omega_{1} \omega_{2}\right\}, \overline{\mathbf{p}}=$ $\left\{\begin{array}{ll}p_{1} & p_{2}\end{array}\right\}^{\mathrm{T}}, a_{j \bar{m} \nu}$ are the known coefficients of the periodic functions, whereas $h_{j \bar{m} \nu}$ are the unknown coefficients of the manifold relation and $e_{j}$ is the $j$ th member of the natural basis, as defined before.

A term-by-term comparison yields an Equation similar to (26). Thus, the 'generalized reducibility condition' can be expressed as

$$
\bar{i}(\overline{\mathbf{p}} \cdot \boldsymbol{\omega})+\sum_{l=1}^{r}\left(m_{l} \lambda_{l}\right)-\bar{\lambda}_{p} \neq 0, \quad \forall v=0, \pm 1, \pm 2, \ldots ; \quad p=1,2, \ldots, s .
$$

It is obvious that if this 'generalized reducibility condition' is satisfied then the vector $\mathbf{H}\left(\mathbf{z}_{r}, t\right)$ can be obtained and the 'slave' states can be expressed in terms of the 'master' states otherwise such an 
order reduction may not be possible. One can investigate the various resonances occurring in the system by studying this 'generalized reducibility condition'. It is to be observed that although the procedure here is discussed for a two-frequency $\left(\omega_{1}, \omega_{2}\right)$ quasiperiodic function $\hat{\mathbf{f}}(\mathbf{x}, t)$, the results are valid for multi-frequency quasiperiodic functions.

\section{An Application}

We apply the order reduction approaches discussed above to a special periodic-quasiperiodic system for which L-F transformation is known in a closed form [11]. Consider a coupled periodic-quasiperiodic nonlinear system given by

$$
\dot{\mathbf{x}}=\overline{\mathbf{A}}(t) \mathbf{x}+\mathbf{f}(\mathbf{x}, t)
$$

where

$$
\overline{\mathbf{A}}=\left[\begin{array}{cccc}
1+\alpha \cos ^{2} t & 1-\alpha \sin t \cos t & 0 & 0 \\
-1-\alpha \sin t \cos t & -1+\alpha \sin ^{2} t & 0 & 0 \\
0 & 0 & 1+\beta \cos ^{2} t & 1-\beta \sin t \cos t \\
0 & 0 & -1-\beta \sin t \cos t & -1+\beta \sin ^{2} t
\end{array}\right]
$$

and

$$
\mathbf{f}(\mathbf{x}, t)=\cos \pi t\left\{x_{1}^{2}+x_{3}^{2} \quad x_{1} x_{2}+x_{3} x_{4} \quad x_{1}^{2}+x_{3}^{2} \quad x_{1} x_{2}+x_{3} x_{4}\right\}^{\mathrm{T}}
$$

For this special system, the L-F transformation is given by [11]

$$
\mathbf{Q}(t)=\left[\begin{array}{cccc}
\cos t & \sin t & 0 & 0 \\
-\sin t & \cos t & 0 & 0 \\
0 & 0 & \cos t & \sin t \\
0 & 0 & -\sin t & \cos t
\end{array}\right]
$$

Applying the L-F transformation $\mathbf{x}=\mathbf{Q}(t) \mathbf{z}$ to Equation (75), we obtain

$$
\dot{\mathbf{z}}=\mathbf{R} \mathbf{z}+\mathbf{Q}^{-1}(t) \mathbf{f}(\mathbf{z}, t) \equiv \mathbf{R} \mathbf{z}+\overline{\mathbf{f}}(\mathbf{z}, t)
$$

where

$$
\mathbf{R}=\left[\begin{array}{cccc}
\alpha-1 & 0 & 0 & 0 \\
0 & -1 & 0 & 0 \\
0 & 0 & \beta-1 & 0 \\
0 & 0 & 0 & -1
\end{array}\right]
$$

and $\overline{\mathbf{f}}(\mathbf{z}, t)$ is a quasiperiodic $(1, \pi)$ vector function with monomials of $z_{i}$. 
Equation (78) can be expressed in the partitioned form according to the eigenvalues of matrix $\mathbf{R}$ and expressed as

$$
\left\{\begin{array}{l}
\dot{\mathbf{z}}_{r} \\
\dot{\mathbf{z}}_{s}
\end{array}\right\}=\left[\begin{array}{cc}
\mathbf{R}_{r} & 0 \\
0 & \mathbf{R}_{s}
\end{array}\right]\left\{\begin{array}{l}
\mathbf{z}_{r} \\
\mathbf{z}_{s}
\end{array}\right\}+\left\{\begin{array}{l}
\overline{\mathbf{f}}_{r}(\mathbf{z}, t) \\
\overline{\mathbf{f}}_{s}(\mathbf{z}, t)
\end{array}\right\}
$$

where $\mathbf{z}_{r}(r \ll r+s)$ are the dominant states to be retained, $\mathbf{z}_{s}$ are the non-dominant states to be eliminated, $\mathbf{R}_{r}$ and $\mathbf{R}_{s}$ are the partitioned matrices corresponding to the dominant and the non-dominant states, respectively, $\overline{\mathbf{f}}_{r}(\mathbf{z}, t)$ and $\overline{\mathbf{f}}_{s}(\mathbf{z}, t)$ are partitioned quasiperiodic vectors of appropriate dimensions.

Once again, the objective of order reduction is to construct an equivalent system of smaller dimension given by

$$
\dot{\mathbf{z}}_{r}=\mathbf{R}_{r} \mathbf{z}_{r}+\overline{\mathbf{f}}_{r}\left(\mathbf{z}_{r}, t\right)
$$

where $\overline{\mathbf{f}}_{r}\left(\mathbf{z}_{r}, t\right)$ is the quasiperiodic nonlinear vector comprising the dominant states. Once the reduced order system is obtained, we can map the results in $\mathbf{x}$ by using the $\mathrm{L}-\mathrm{F}$ transformation.

\subsection{ORDER REDUCTION USING THE LINEAR APPROACH}

With $\alpha=0.7$ and $\beta=0.3$ Equation (79) yields

$$
\left\{\begin{array}{l}
\dot{z}_{1} \\
\dot{z}_{2} \\
\dot{z}_{3} \\
\dot{z}_{4}
\end{array}\right\}=\left[\begin{array}{cccc}
-0.3 & 0 & 0 & 0 \\
0 & -1 & 0 & 0 \\
0 & 0 & -0.7 & 0 \\
0 & 0 & 0 & -1
\end{array}\right]\left\{\begin{array}{l}
z_{1} \\
z_{2} \\
z_{3} \\
z_{4}
\end{array}\right\}+\left\{\begin{array}{l}
\bar{f}_{1}\left(z_{1}, z_{2}, z_{3}, z_{4}, t\right) \\
\bar{f}_{2}\left(z_{1}, z_{2}, z_{3}, z_{4}, t\right) \\
\bar{f}_{3}\left(z_{1}, z_{2}, z_{3}, z_{4}, t\right) \\
\bar{f}_{4}\left(z_{1}, z_{2}, z_{3}, z_{4}, t\right)
\end{array}\right\}
$$

We choose $z_{1}$ and $z_{2}$ as the modes to be retained and neglect the entire contribution from the states $z_{3}$ and $z_{4}$. Thus, the linear reduced order system is given by

$$
\left\{\begin{array}{l}
\dot{z}_{1} \\
\dot{z}_{2}
\end{array}\right\}=\left[\begin{array}{cc}
-0.3 & 0 \\
0 & -1
\end{array}\right]\left\{\begin{array}{l}
z_{1} \\
z_{2}
\end{array}\right\}+\left\{\begin{array}{l}
\bar{f}_{1}\left(z_{1}, z_{2}, 0,0, t\right) \\
\bar{f}_{2}\left(z_{1}, z_{2}, 0,0, t\right)
\end{array}\right\}
$$

The system given by Equation (82) is numerically integrated with typical initial conditions and the results are mapped in $\mathbf{x}$ domain by using the $\mathrm{L}-\mathrm{F}$ transformation. The time traces are given in Figure 8a. The time trace of reduced order system is compared with the time trace obtained from integration of large-scale system given by Equation (75). It can be seen that the time traces are in good agreements. Further, the phase plots (given by Figure 8b) are also compared and they are in good agreement. As the system is asymptotically stable, it attains the equilibrium after a certain period. 


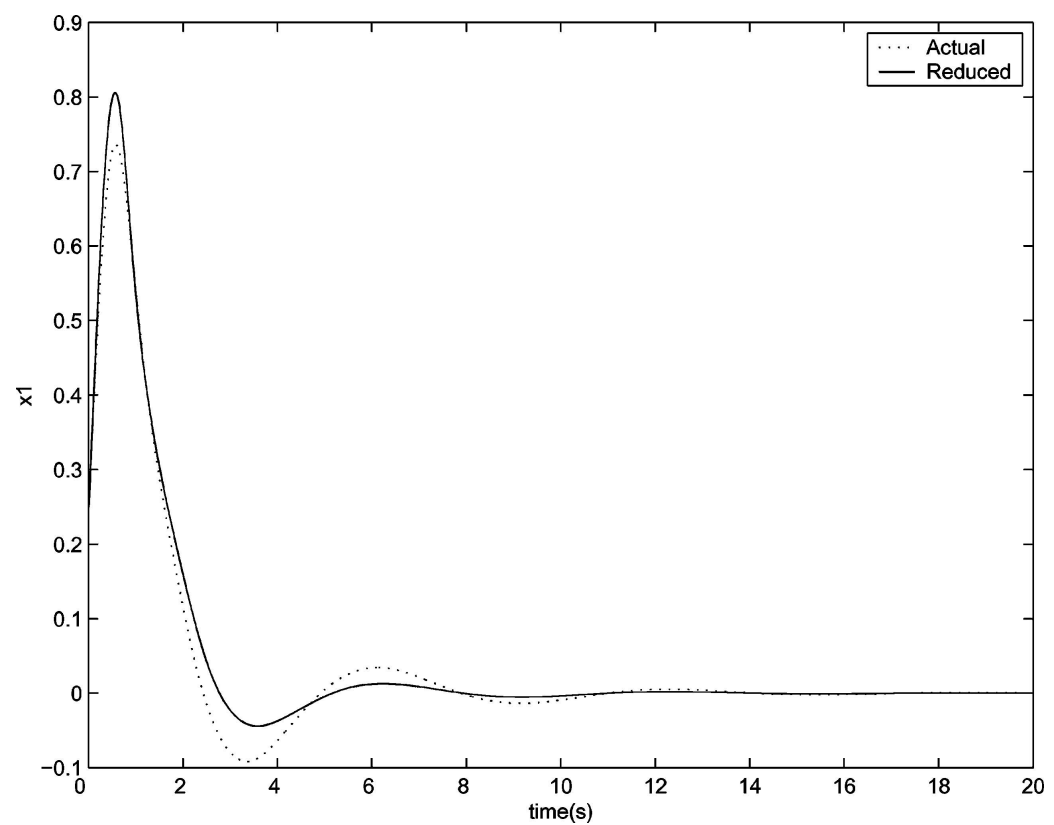

(a)

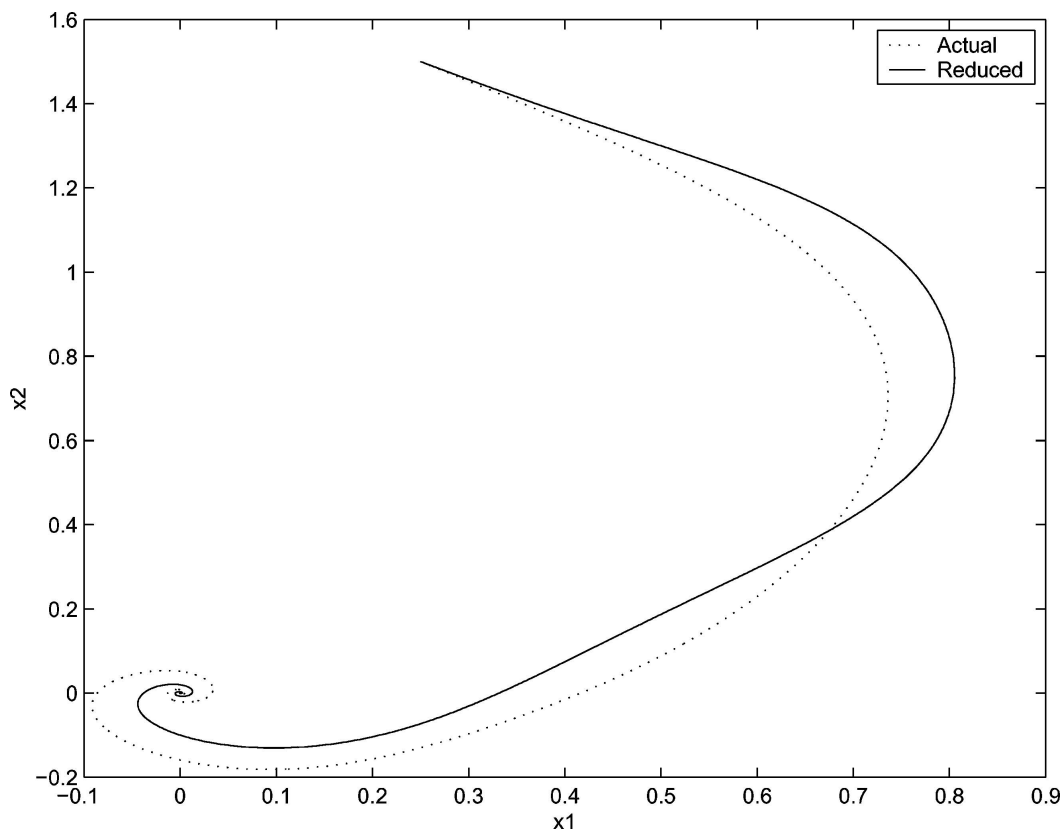

(b)

Figure 8. (a) Time trace of actual and reduced order system using linear method. (b) Phase portrait of actual and reduced order system using linear method. 


\subsection{Order Reduction Using the Singular Perturbation ApProach}

If the derivatives of the non dominant states $\left(z_{3}, z_{4}\right)$ in Equation (81) are set equal to zero, the resulting DAE system is given by

$$
\left\{\begin{array}{c}
\dot{z}_{1} \\
\dot{z}_{2} \\
0 \\
0
\end{array}\right\}=\left[\begin{array}{cccc}
-0.3 & 0 & 0 & 0 \\
0 & -1 & 0 & 0 \\
0 & 0 & -0.7 & 0 \\
0 & 0 & 0 & -1
\end{array}\right]\left\{\begin{array}{l}
z_{1} \\
z_{2} \\
z_{3} \\
z_{4}
\end{array}\right\}+\left\{\begin{array}{l}
\bar{f}_{1}\left(z_{1}, z_{2}, z_{3}, z_{4}, t\right) \\
\bar{f}_{2}\left(z_{1}, z_{2}, z_{3}, z_{4}, t\right) \\
\bar{f}_{3}\left(z_{1}, z_{2}, z_{3}, z_{4}, t\right) \\
\bar{f}_{4}\left(z_{1}, z_{2}, z_{3}, z_{4}, t\right)
\end{array}\right\}
$$

This system is solved numerically using Mathematica-5 ${ }^{\mathbf{T M}}$ and after the L-F transformation results in $\mathbf{x}$ domain are obtained. These results are compared with those obtained by integrating large-scale system given by Equation (75). Again, the time traces (Figure 9a) and the phase plots (Figure 9b) are compared and they are in better agreement compared to the linear approach.

Alternatively, the approximate solution of Equation (83) may be obtained using the fixed-point iteration method and we have

$$
\left\{\begin{array}{c}
z_{3} \\
z_{4}
\end{array}\right\}=\left\{\begin{array}{c}
\bar{f}_{3}\left(z_{1}, z_{2}, 0,0, t\right) /(-0.7) \\
\bar{f}_{4}\left(z_{1}, z_{2}, 0,0, t\right) /(-1)
\end{array}\right\}
$$

Further refinement in this approximation is possible by using a second iteration. It is found that this refinement does not yield significant increase in accuracy of the solution and hence not pursued here.

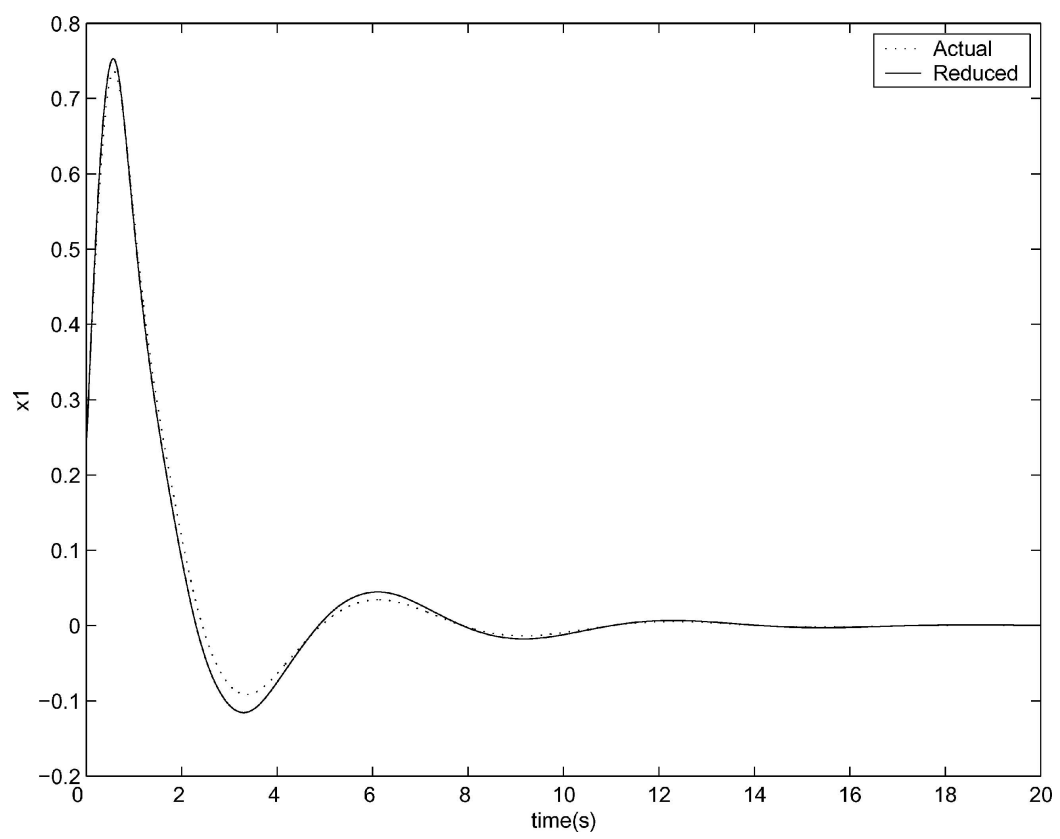

(a)

Figure 9. (a) Time trace of actual and reduced order system using SP (direct) method. (b) Phase portrait of actual and reduced order system using SP (direct) method. 


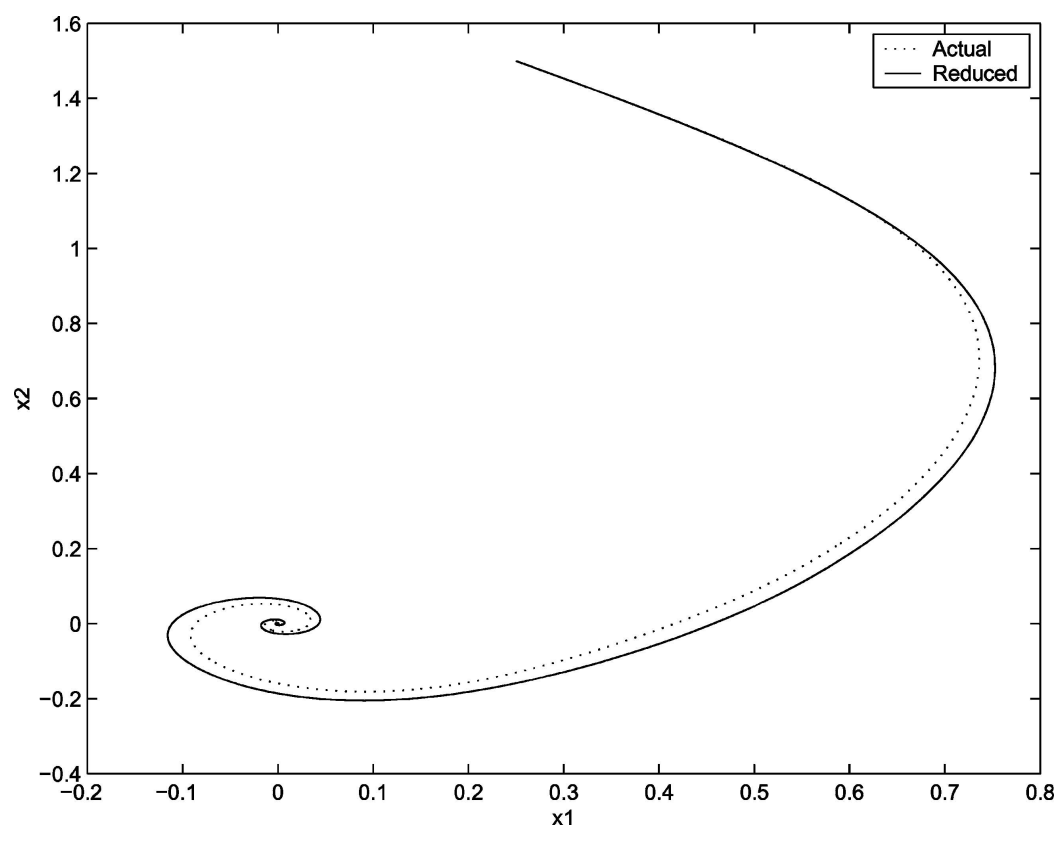

(b)

Figure 9. (Continued.)

Thus, the reduced order Equation is obtained by substituting Equation (84) in the top part of ('master' dynamics) Equation (83) and a reduced order model in a closed form is obtained as

$$
\left\{\begin{array}{l}
\dot{z}_{1} \\
\dot{z}_{2}
\end{array}\right\}=\left[\begin{array}{cc}
-0.3 & 0 \\
0 & -1
\end{array}\right]\left\{\begin{array}{l}
z_{1} \\
z_{2}
\end{array}\right\}+\left\{\begin{array}{l}
\bar{f}_{1}\left(z_{1}, z_{2}, \bar{f}_{3}\left(z_{1}, z_{2}, 0,0, t\right) /(0.3-1),-\bar{f}_{4}\left(z_{1}, z_{2}, 0,0, t\right), t\right) \\
\bar{f}_{2}\left(z_{1}, z_{2}, \bar{f}_{3}\left(z_{1}, z_{2}, 0,0, t\right) /(0.3-1),-\bar{f}_{4}\left(z_{1}, z_{2}, 0,0, t\right), t\right)
\end{array}\right\}
$$

It is worth pointing out that the order of nonlinearity in Equation (85) is more that that of the original Equation. As before, Equation (85) is integrated numerically and using the L-F transformation all states in $\mathbf{x}$ can be obtained. Again, the time traces (Figure 10a) and the phase plots (Figure 10b) of the reduced order system are compared with the large-scale system and they show improvement over the linear approach.

Let us consider a special case when $\beta=1$ : it can be seen that Equation (84) blows up yielding no solution. This is similar to the 'parametric resonance' case (zero Floquet exponents) discussed in the previous section. This implies that the resonating modes (corresponding to the zero Floquet exponents) must be chosen as 'master' modes for an accurate order reduction.

\subsection{ORDER REDUCTION USING THE POST-PROCESSING}

Post processing is a way to correct the linear solution by considering the nonlinear effects. As before, the post-processing correcting factor $\boldsymbol{\kappa}\left(\mathbf{z}_{r}, t\right)$ is obtained by solving bottom part of Equation (83) iteratively. We follow the single point iteration and obtain $\kappa\left(\mathbf{z}_{r}, t\right)$ as discussed before and the solution obtained 


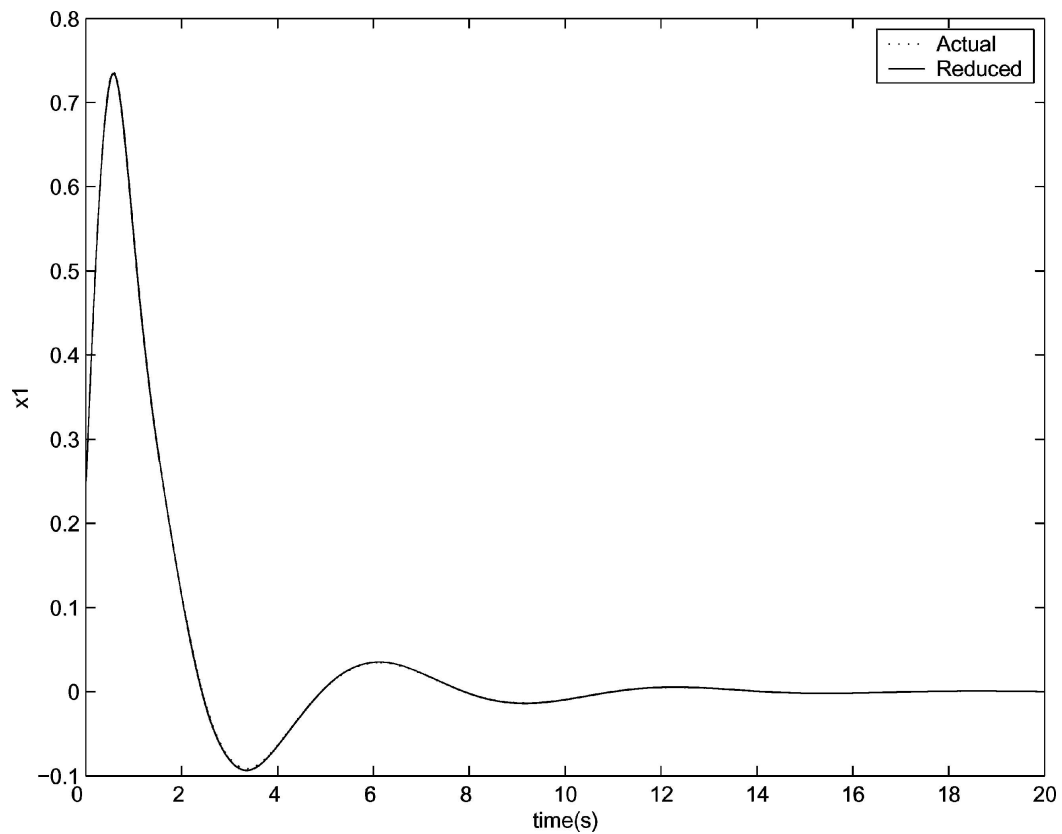

(a)

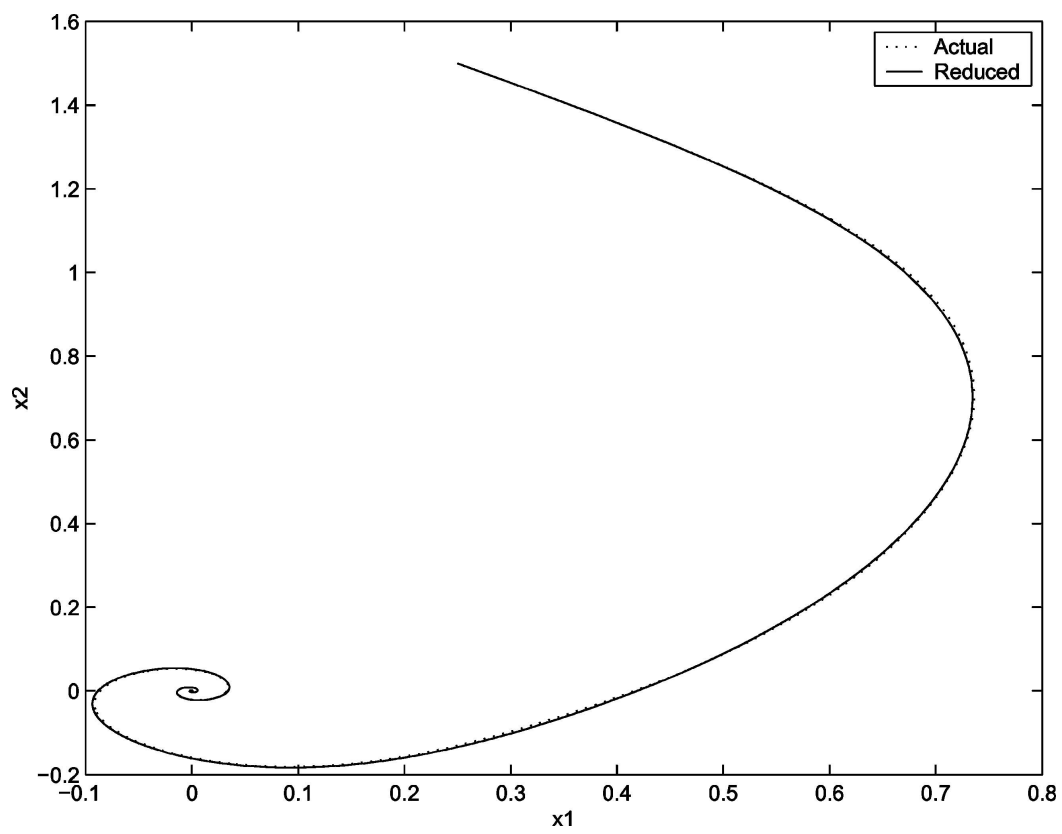

(b)

Figure 10. (a) Time trace of actual and reduced order system using SP (iteration) method. (b) Phase portrait of actual and reduced order system using SP (iteration) method. 
by integrating Equation (82) is 'uplifted' as

$$
\left\{\begin{array}{l}
\bar{z}_{1} \\
\bar{z}_{2}
\end{array}\right\}=\left\{\begin{array}{l}
z_{1} \\
z_{2}
\end{array}\right\}+\left\{\begin{array}{l}
\kappa_{3}\left(z_{1}, z_{2}, t\right) \\
\kappa_{4}\left(z_{1}, z_{2}, t\right)
\end{array}\right\}
$$

The obvious advantage here is the saving in the computation time as the post-processing factor can be found out separately and does not depend upon initial conditions. This method relies on the linear solution and cannot yield meaningful results if the linear approach fails or if the initial conditions are large. As before, the solution in $\mathbf{x}$ domain can be obtained by transforming $\left\{\bar{z}_{1} \bar{z}_{2}\right\}^{\mathrm{T}}$ using the L-F transformation. For comparison purposes, the time traces and the phase plots of the reduced order system and the large-scale system are given in Figures 11a and b, respectively. It can be seen that for small initial conditions the results match much better that the results obtained from the liner approach. This method is more useful when the limited computational resources prohibit the use of singular perturbation approach or the invariant manifold approach and the linear order reduction technique does not provide results within the acceptable range even for small initial conditions.

\subsection{ORDER REDUCTION USING THE INVARIANT MANIFOLD APPROACH}

Here the nonlinear relationship between the non-dominant states and the dominant states is assumed to be quasiperiodic. If the system does not exhibit 'time dependent or time independent resonances', (like the case under consideration) then the 'generalized reducibility condition' given by Equation (74) is satisfied and the system order can be reduced.

We start with Equation (81) and select the same states $\left[\mathbf{z}_{r}=\left\{z_{1} z_{2}\right\}^{\mathrm{T}}\right]$ as the dominant states which correspond to the lower system eigenvalues and try to find a nonlinear quasiperiodic relationship of the form given in Equation (70). For this particular example, the relationship between $\mathbf{z}_{s}$ and $\mathbf{z}_{r}$ are given by

$$
\mathbf{z}_{s}=\sum_{i} \mathbf{h}_{i}\left(z_{1}, z_{2}, t\right) \equiv \mathbf{H}\left(z_{1}, z_{2}, t\right), \quad s=3,4
$$

where

$$
\mathbf{h}_{i}=\sum_{\overline{\mathbf{m}}} \overline{\mathbf{h}}_{i}(t) z_{1}^{m_{1}} \ldots z_{2}^{m_{2}}, \quad \overline{\mathbf{m}}=\left(m_{1}, m_{2}\right)^{\mathrm{T}}, \quad m_{1}+m_{2}=2, \quad m_{1}, m_{2}=0,1,2
$$

Here $\overline{\mathbf{h}}_{i}(t)$ are the unknown quasiperiodic vector coefficients. We substitute Equation (87) into Equation (78) and after expanding $\overline{\mathbf{h}}_{i}(t)$ and $\mathbf{w}_{s}\left(z_{r}, t\right)(s=3,4)$ in double Fourier series and neglecting the terms of higher order, we obtain the relationship between the dominant and the non-dominant states as

$$
\begin{aligned}
H_{3}= & (1.02 \cos t \cos \pi t+0.99 \cos \pi t \sin \pi t+0.31 \cos t \sin \pi t) z_{1}^{2} \\
& +(0.73 \cos t \cos \pi t+0.14 \cos \pi t \sin t+0.30 \sin t \sin \pi t) z_{1} z_{2} \\
H_{4}= & (-0.24 \cos t \cos \pi t+0.91 \cos \pi t \sin t+0.31 \cos t \sin \pi t) z_{1} z_{2} \\
& +\left(\left(-\frac{1}{8}+\frac{\bar{i}}{8}\right)(1+\cos 2 \pi t+\bar{i} \sin 2 \pi t)(\cos (t-\pi t)+\bar{i} \sin (t-\pi t))\right. \\
& \left.-\left(\frac{1}{8}+\frac{\bar{i}}{8}\right)(1+\cos 2 \pi t+\bar{i} \sin 2 \pi t)(\cos (t+\pi t)-\bar{i} \sin (t+\pi t))\right) z_{2}^{2}
\end{aligned}
$$




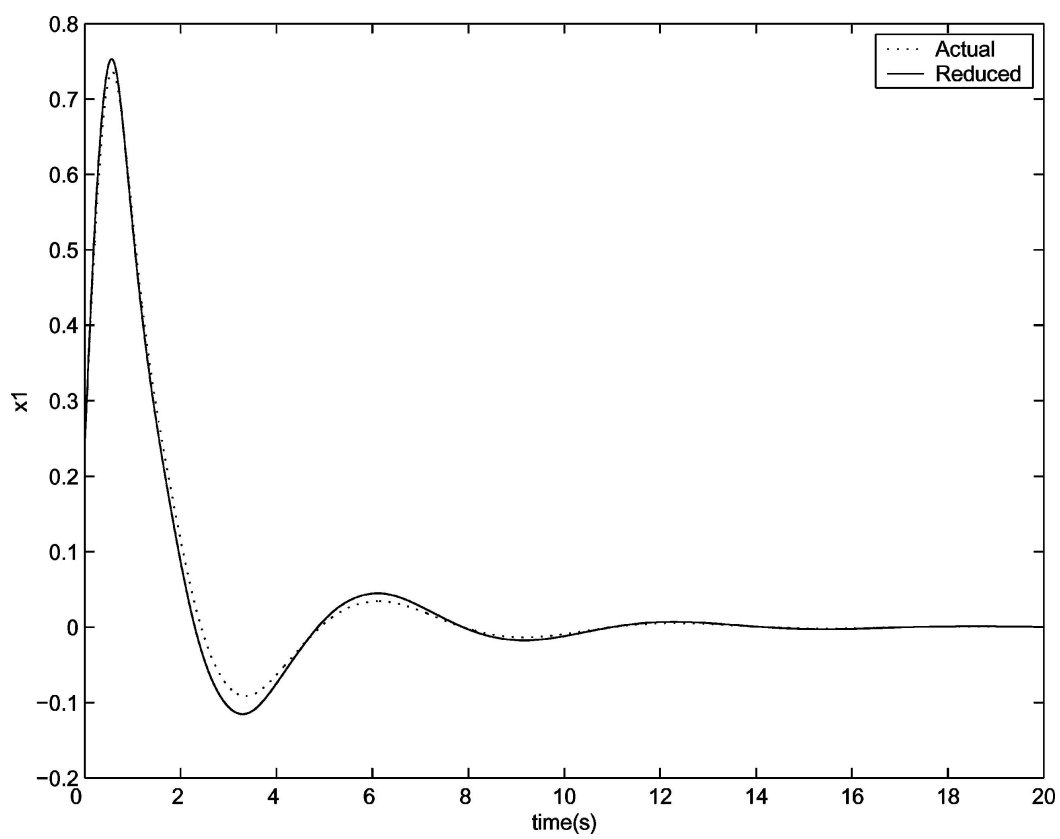

(a)

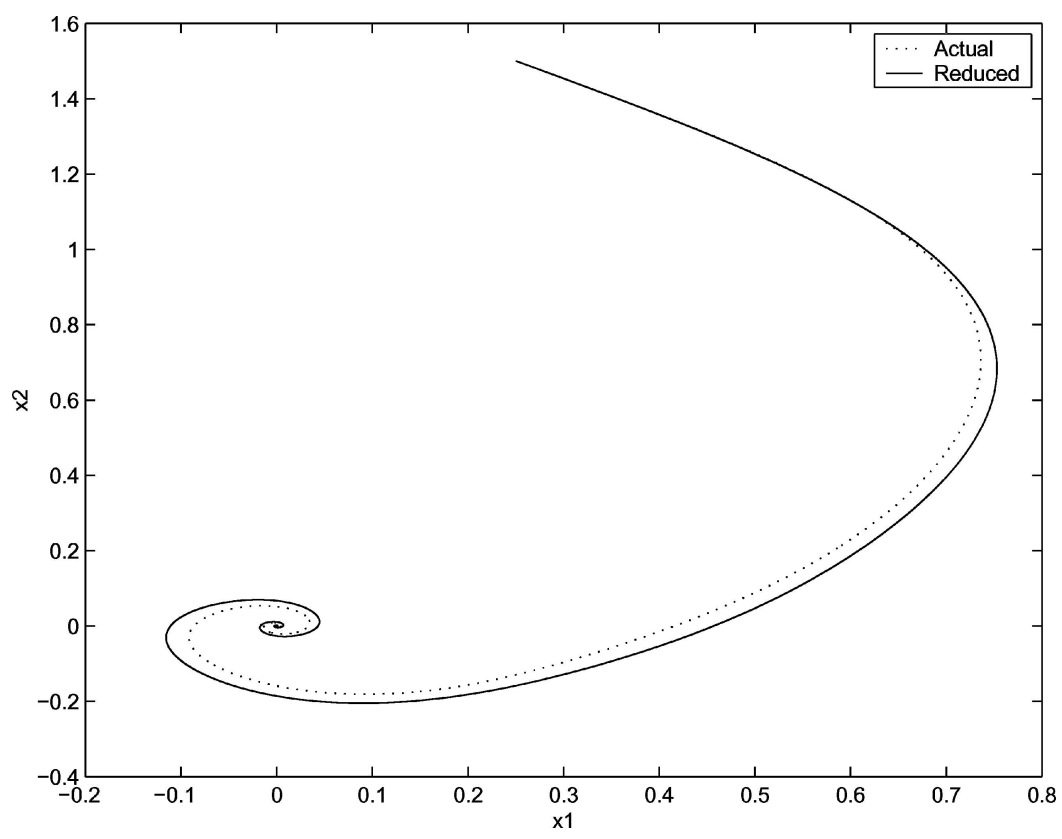

(b)

Figure 11. (a) Time trace of actual and reduced order system using post-processing method. (b) Phase portrait of actual and reduced order system using post-processing method. 
270 S. C. Sinha et al.

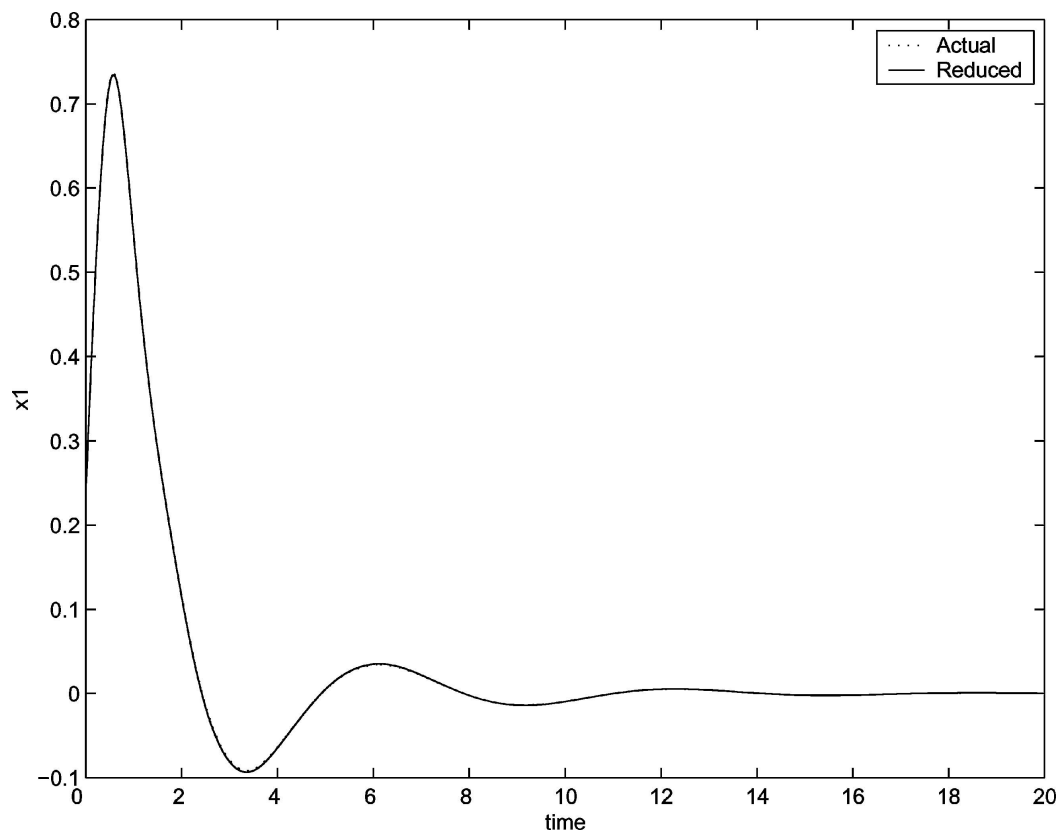

(a)

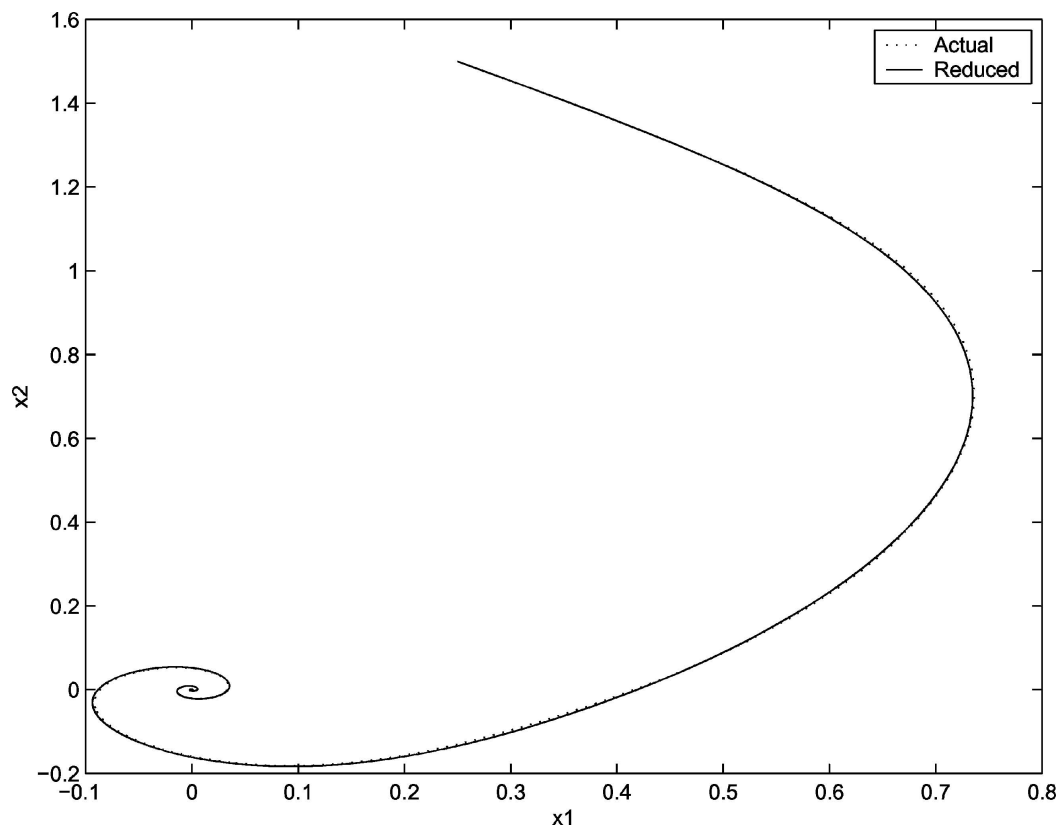

(b)

Figure 12. (a) Time trace of actual and reduced order system using inv. manifold method. (b) Phase portrait of actual and reduced order system using invariant manifold method. 


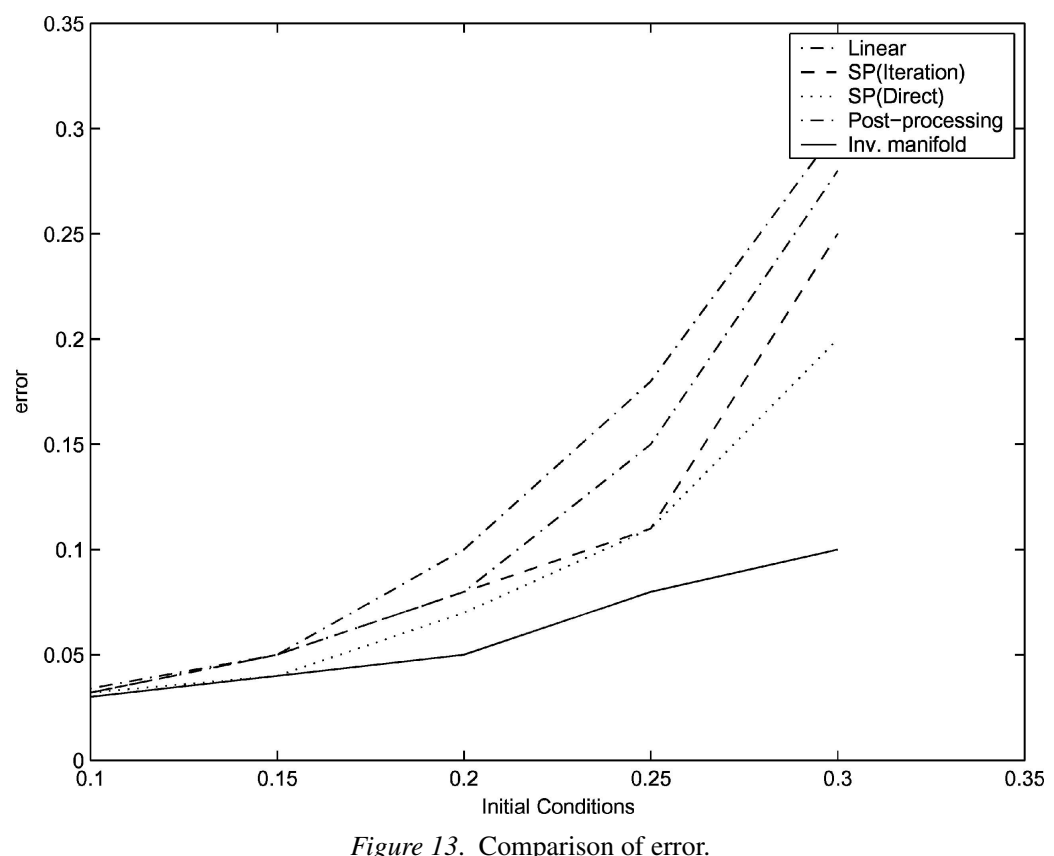

Equations (89) and (90) are substituted into the top half of Equation (81) to obtain the reduced order model as

$$
\left\{\begin{array}{l}
\dot{z}_{1} \\
\dot{z}_{2}
\end{array}\right\}=\left[\begin{array}{cc}
-0.3 & 0 \\
0 & -1
\end{array}\right]\left\{\begin{array}{l}
z_{1} \\
z_{2}
\end{array}\right\}+\left\{\begin{array}{l}
\bar{f}_{1}\left(z_{1}, z_{2}, H_{3}\left(z_{1}, z_{2}, t\right), H_{4}\left(z_{1}, z_{2}, t\right), t\right) \\
\bar{f}_{2}\left(z_{1}, z_{2}, H_{3}\left(z_{1}, z_{2}, t\right), H_{4}\left(z_{1}, z_{2}, t\right), t\right)
\end{array}\right\}
$$

This symbolic computation was carried out using Mathematica ${ }^{\mathrm{TM}}$. Equation (91) was integrated numerically to determine response of the reduced order system. Afterwards by using the L-F transformation, the results in $\mathbf{x}$ domain are obtained.

For comparison purposes, the time traces of the reduced order model and the large-scale model are presented in Figure 12a. It can be seen that they match extremely well. The phase portraits given by Figure $12 \mathrm{~b}$ also match very well. Thus, it can be concluded that the invariant manifold based approach yields the most accurate reduced order model. As before, in order to get a global perspective, error norm associated with various methodologies is plotted as a function of initial conditions in Figure 13. It can be seen that the invariant manifold approach is the most accurate and the linear approach is the least accurate.

\section{Discussion and Conclusions}

In this paper, order reduction techniques for parametrically excited nonlinear systems are presented. The order reduction can be carried out either in state space or in second order (structural) form. For reducing order in the state space, four techniques are proposed. All techniques make use of the L$\mathrm{F}$ transformation that produces equivalent systems with time invariant linear parts and time-periodic nonlinear parts.

The first procedure is a linear technique that may be considered as a state space version of 'Guyan Reduction' where the entire non-dominant dynamics is neglected. As an improvement over the linear 


\section{S. C. Sinha et al.}

technique, a 'nonlinear projection method', based on singular perturbation, is suggested. Here, one can directly integrate the resulting system of DAEs or use some fixed-point iteration techniques. In order to reduce the computational cost, a post-processing method is also suggested. The 'invariant manifold technique' is a nonlinear order reduction methodology similar to the concept of 'time periodic center manifold theory'. It yields a 'reducibility condition' that determines whether (or not) an order reduction is possible. It is an extremely important result because it provides correct definitions for 'true internal resonance' and 'true combination resonance' in parametrically excited nonlinear systems in terms of Floquet exponents. Under the assumptions that the parametric excitation terms are small, traditionally well-known conditions for 'internal resonance' in autonomous nonlinear systems and 'combination resonance' in time-periodic nonlinear systems are recovered. Reduced order models constructed from this method capture the dynamics of original large-scale system more accurately than other approaches.

An alternate methodology for obtaining reduced order models of nonlinear time periodic systems represented by a set of second order equations is also proposed. The systems considered for order reduction are not limited by the norm or the strength of the periodic terms. The 'reducibility (or resonance) conditions' in this case are also derived using a novel approach. A practical example depicting the procedure is also included.

The methodologies proposed for order reduction in state space can be generalized to reduce the order of a special class of systems known as periodic-quasiperiodic systems. All techniques, except the invariant manifold approach can be used in a straightforward manner for constructing the reduced order models. In order to apply the invariant manifold approach, a nonlinear quasiperiodic relationship is assumed that yields a 'generalized reducibility condition'. The comparison of time traces, phase portraits and error analysis shows that the reduced order model obtained by the invariant manifold approach yields the most accurate results, the linear approach produces the least accurate results and the outcomes from other techniques fall in between.

In conclusion, it can be stated that some practical techniques for order reduction of general nonlinear time periodic systems undergoing strong parametric excitation have been suggested. Mathematical conditions are also derived to determine whether (or not) a large-scale nonlinear parametrically excited system can be reduced to a lower order system. Several extensions, generalizations and applications to control problems are in progress.

\section{Acknowledgements}

Financial support provided by the Air Force Office of Scientific Research (AFOSR) in collaboration with the University of Alaska Fairbanks under the contract no. F49620-01-1-0388 and National Science Foundation (contract no. CMS 0114571) is gratefully acknowledged.

\section{References}

1. Mahmoud, M. S. and Singh, M. G., Large Scale Systems Modelling, Pergamon Press, Oxford, UK, 1981.

2. Guyan, R. J., 'Reduction of stiffness and mass matrices', AIAA Journal 3, 1965, 380.

3. Shokoohi, S., Silverman, L., and Van Dooren, P., 'Linear time-variable systems: Balancing and model reduction', IEEE Transactions on Automatic Control AC-28, 1983, 810-822.

4. Shaw, S. W. and Pierre, C., 'Nonlinear normal modes and invariant manifolds', Journal of Sound and Vibration 150(1), 1991, 170-173. 
5. Vakakis, A. F., 'Non-linear normal modes (NNMs) and their applications in vibration theory: An overview', Mechanical Systems and Signal Processing 11(1), 1997, 3-22.

6. Shaw, S. W., Pierre, C., and Pesheck, E., 'Modal analysis-based reduced-order models for nonlinear structures - an invariant manifold approach', The Shock and Vibration Digest 31(1), 1999, 3-16.

7. Burton, T. D. and Young, M. E., 'Model reduction and nonlinear normal modes in structural dynamics', in Proceedings of ASME Winter Annual Meeting, Chicago, IL, 1994.

8. Burton, T. D. and Rhee, R. W., 'The reduction of nonlinear structural dynamic models,' Journal of Vibration and Control 6(4), 2000, 531-556.

9. Gallivan, K., Grimme, E., and Van Dooren, P., 'Multi-point Padé approximants of large scale system via two-sided rational Krylov algorithm', in Proceedings of the IEEE Conference on Decision and Control, Lake Buena Vista, FL, 1994.

10. Guilhen, P. M., Bertheier, P., Ferraris, G., and Lalanne, M., 'Dynamic behavior of dissymmetric rotor bearings modelled periodic coefficient large system', in Proceedings of 58th Shock and Vibration Symposium, Huntsville, AL, 1987.

11. Sinha, S. C. and Pandiyan, R., 'Analysis of quasilinear dynamical systems with periodic coefficients via Liapunov-Floquet Transformation', International Journal of Non-Linear Mechanics 29, 1994, 687-702.

12. Sinha, S. C., Pandiyan, R., and Bibb, J. S., 'Liapunov-Floquet transformation: Computation and applications to periodic systems', ASME Journal of Vibration and Acoustics 118, 1996, 209-219.

13. Deshmukh, V., Sinha, S. C., and Joseph, P., 'Order reduction and control of parametrically excited dynamical systems', Journal of Vibration and Control 6, 2000, 1017-1028.

14. Sinha, S. C., Redkar, S., and Butcher, E., 'Order reduction of nonlinear time periodic coefficients using invariant manifolds', Journal of Sound and Vibration, 2005 (in press).

15. Deshmukh, V., Butcher, E., and Sinha, S. C., 'Order reduction of parametrically excited nonlinear structural systems via invariant manifolds', in review Journal of Vibration and Acoustics, 2004.

16. Arnold, V. I., Ordinary Differential Equations, MIT Press, Boston, MA, 1978.

17. Wooden, S., Application of Lyapunov-Floquet Transformation and Quasiperiodic Normal Forms to Dynamical Systems, MS thesis, Auburn University, Auburn, AL, 2002.

18. Yakubovich, V. A. and Starzhinski, V. M., Linear Differential Equations with Periodic Coefficients, Vols. 1 and 2, Wiley, New York, 1975.

19. Kokotović, P., Khalil, H., and O’Reilly, J., Singular Perturbation Methods in Control Analysis and Design, SIAM Press, Philadelphia, PA, 1999.

20. Kan, D., Nonlinear Galerkin Based Model Reduction, Technical Report No. (98-49), Department of Mathematics, UCLA, Los Angeles, CA, 1998.

21. Redkar, S., Sinha, S. C., and Butcher, E., 'Some techniques for order reduction of nonlinear time periodic systems', in Proceedings of IMECE 2003, Washington DC, November 15-21, 2003, Paper No. 42552.

22. Steindl, A. and Troger, H., 'Methods for dimension reduction and their application in nonlinear dynamics', International Journal of Solids and Structures 38, 2001, 2131-2147.

23. Sinha, S. C., Redkar, S., and Butcher, E., 'On macromodeling of nonlinear systems with time periodic coefficients', Communications in Nonlinear Science and Numerical Simulation, 2005 (in press).

24. Garcia-Archilla B., Novo, J., and Titi, E., 'Postprocessing the Galerkin method: A novel approach to approximate inertial manifolds', SIAM Journal on Numerical Analysis 35(3), 1998, 941-958.

25. Evan-Iwanowski, R. M., Resonance Oscillations in Mechanical Systems, Elsevier, Amsterdam, The Netherlands, 1976.

26. Hsu, C. S., 'On the parametric excitation of a dynamic system having multiple degree of freedom', Journal of Applied Mechanics 30, 1963, 367-372.

27. Malkin, I. G., Some Problems in the Theory of Nonlinear Oscillations (Translated from Russian), State Publishing House of Technical and Theoretical Literature, Moscow, 1956.

28. Sinha, S. C., Redkar, S., Butcher, E., and Deshmukh, V., 'Order reduction of nonlinear time periodic systems using invariant manifolds', Paper no. VIB-48445, in Proceedings of 19th Biennial Conference on Mechanical Vibration and Noise, Chicago, IL, September 2-6, 2003.

29. Sinha, S. C., Butcher, E. A., and Dávid, A., 'Construction of dynamically equivalent time-invariant forms for time-periodic systems', Nonlinear Dynamics 16, 1998, 203-221. 